\title{
Who Quits Next? \\ Firm Growth in Growing Economies
}

\author{
Julieta Caunedo \\ Emircan Yurdagul* \\ Cornell University Washington U. in St. Louis
}

February 13, 2015

\begin{abstract}
This paper provides a theory linking characteristics of the industry dynamics to aggregate growth. We analyze firms' life cycle productivity, employment-age profiles, and firm selection across countries. Using a large cross-country dataset we document (i) more frequent labor productivity growth for firms operating in fast growing economies, (ii) lack of systematic relationship between the tail of the employment size distribution and growth and (iii) steeper employment-age profiles in slow growing economies. Our working thesis is that firms' likelihood of turning their investments into actual productivity growth, and uncertainty on their returns if successful, impacts firms investment in productivity, selection and aggregate growth. We think of firm uncertainty broadly, to include for example political instability, changes in tax regimes, lack of social capital or firm demand fluctuations. We argue that in slow growing rich productive economies, steep-employment age profiles are related to high return uncertainty and strong firm selection. We are able to accommodate poor and rich slow growing economies by decoupling firm's probability of success from return uncertainty. We build a tractable general equilibrium model that displays endogenous long run growth compatible with a stationary size distribution and the documented empirical facts. We contribute to the literature by analyzing how variations in the probability of firm success and return uncertainty account for differences in observed industry structure and its relationship with aggregate growth.
\end{abstract}

Keywords: Productivity, Firm Selection, Economic Growth. JEL codes: E23, O4.

\footnotetext{
*Contact: julieta.caunedo@cornell.edu or yurdagul@wustl.edu
} 


\section{Introduction}

Entrepreneurs, in braving uncertainty, face the risk of being replaced if their ventures are not sufficiently successful given the performance of other firms in the market. Selection and return uncertainty are key in determining the characteristics of firms operating in the market and growth of the economy. Economies where firms invest little in productivity and grow slower may have lower growth not only because the levels of investment are low, but also because the process of firm selection in the market slows down. Entrepreneurs face two different layers of uncertainty: a) on whether a given outcome is realized (i.e. introduction of a new product to the market); but also, conditional on success, b) on the return to any given investment (i.e. profits associated to the new product in the market). Often times, ventures that entail high uncertainty in both dimensions, such as those associated to disruptive technologies, are the ones pushing growth the most. ${ }^{1}$

In this paper, we argue that disparities in the probability with which firms turn their investments into actual labor productivity growth (probability of success hereafter), and uncertainty on returns if successful, can explain the disparate behavior of firm growth and selection across economies. Then, we show how these two determine of aggregate growth. We show that patterns of industry dynamics such as the number of operating firms in an industry, employment-age profiles and age distribution hold information about both dimensions of firm uncertainty. Both of which are quantitatively relevant in explaining differences in aggregate growth across countries.

We start by describing cross-country patterns of aggregate growth and industry dynamics: establishments' labor productivity growth (productivity growth hereafter) and employment and age distributions. We use the 2006-2011 Enterprise Survey data by the World Bank. We analyze their relationship with aggregate GDP growth and

\footnotetext{
${ }^{1}$ See Christensen (1997) for a detailed description disruptive technologies.
} 
income per capita. We document (i) higher frequency of firm level productivity growth ${ }^{2}$ in countries with higher real gdp growth, (ii) no systematic differences in the tail of the employment size distribution across countries with different aggregate growth ${ }^{3}$, and (iii) steeper employment-age profiles in slow growing economies.

Fact (i) is to be expected if aggregate growth is connected to the activity of the firms operating in the market. Facts (ii) and (iii) are new and less understood. This paper sheds light on simple mechanisms that may explain both.

We interpret the lack of systematic relationship between growth and the top shares of the employment distribution as a symptom of the characteristics of economic growth. If selection in the market is related to the pace of aggregate economic growth, the growth rate of the threshold productivity level for survival would likely reflect aggregate growth of the economy. If firms grow fast, survival thresholds increase accordingly and aggregate growth can be high. When large old firms are relatively unproductive, firm growth is slow, selection is damaged and small unproductive firms can survive longer, while aggregate growth is low. In either case, the share of employment accounted for by the largest firms in the economy, relative to the average firm, can be the same.

Steep employment-age profiles in slow growth economies are at odds with a pure selection theory. Such theory predicts positive correlation between selection and the speed of growth both at the firm level and in the aggregate. Two issues arise. First, the data analysis is cross sectional, so these profiles cannot be interpreted as firm employment growth paths necessarily (longitudinal data). Instead, they reflect the fact that in slow growing economies, relatively large older firms coexist with small young ones. Second, aggregate growth in the economy is related to the pace of average productivity improvements, whereas firm employment growth, reflects firm productivity improvements relative to the mean. There are two types of slow growing economies: very unproductive poor ones, and very productive rich ones. In the former, fact (iii)

\footnotetext{
${ }^{2}$ after controlling for average growth in the economy

${ }^{3}$ although, in line with the literature, there is a negative correlation with gdp per capita
} 
is consistent with weak selection, which allows small unproductive firms to survive, and coexist with large slow growth firms. In the latter, fact (iii) is related to strong selection, young small firms entering and exiting the market but sometimes experiencing high productivity growth relative to the mean. If the those successful firms are relatively few, average output growth is low.

To understand the relationship between the probability of success and return uncertainty and the highlighted empirical facts, we build a tractable model with endogenous firm selection. In our benchmark, firms are allowed to invest to grow but whether the returns to investment are realized or not is uncertain. In this benchmark, no additional return uncertainty is allowed. There is a continuum of firms. Firms can be of the innovative type, or they can just operate with a constant productivity technology. At the beginning of each period, innovative firms decide whether or not to invest in technology improvements and how much to produce in the current period. If they invest, they pay a cost that is a non-linear function of their investment and get returns one period ahead with some probability. The probability of success is the same for all the firms in the market, but the realizations of course need not be. Innovative firms can also decide to liquidate, at which point their technology is taken over by a non-innovative firm. Non-innovative firms decide whether to stay in the market, produce and pay operating costs, or exit.

The key fundamental difference across economies is the probability of realizing returns to investment. Real life factors that would affect this rate include political instability, changes in tax regimes, changes in terms of trade. These factors have a direct impact on the life cycle of firms through the frequency of growth episodes. In particular, firms would have different number of periods with positive growth. Probability of success also alters the expected return per dollar invested, and hence the size of those investments in equilibrium. Together, these two channels will generate firm productivity growth profiles that are very different across economies. We analyze a general equilibrium model, which is critical for our purposes for two reasons. First, in an economy 
with higher probability of positive returns to investment, firms' productivity growth would be faster; which implies that relatively unsuccessful firms exit quicker (strong selection). Hence, growth in this economy will be higher than the level predicted by a simple partial equilibrium model where selection is not accounted for. Second, as long as the advantage of an economy in terms of the returns to investment is common to all of its firms, the allocation of employment and sales among those is not necessarily different from an economy with a lower probability of success. This helps our model replicate the absence of a systematic relationship between growth and the allocation of employment across firms.

Preliminary computations show that variations in the probability of success that firms face have quantitative impact on both the rate of investment at the firm level and aggregate growth of the economy. Moreover, the share of firms investing in productivity growth, as well as the equilibrium measure of firms operating in the market changes with the underlying probability of success. This holds even for processes that generate the same volatility of revenues ex post. More importantly, we generate equivalent tails of the employment size distribution across economies.

Without return uncertainty, the existence of a balance growth path poses tight restrictions on equilibrium productivity growth by the firms operating in the market. In particular, there is no rank reversal, which in turn implies no employment growth. Intuitively, firms' productivity grows at a constant rate along the balance growth path. If the firm is successful, it grows at everybody's rate. Hence, its employment (which depends on its relative productivity vis a vis the average) does not change. If instead the firm is unsuccessful, employment shrinks, as it is now relatively less productive than before. When we augment our benchmark economy to allow return uncertainty (and hence for rank reversals), we show that the probability of success jointly with firm uncertainty are relevant to determine the relationship between employment-age profiles and firms' age distribution with aggregate growth in the economy. Furthermore, return uncertainty and success probability jointly determine the pace of selection in the market 
and the decoupling between average firm growth and aggregate growth.

Related literature. This paper provides a theory that links characteristics of the industry dynamics to aggregate growth, consistently with the patterns documented in the data. Allocation of factors across firms has been shown to be key in understanding aggregate productivity differences across countries, and through them, income per capita (Hsieh and Klenow (2009), Restuccia and Rogerson (2008)). Until recently, most of the literature that studies the link between the micro structure of the economy and productivity focused on static allocations. However, static allocations can reflect firm level distortions that can have lasting effects on incentives to innovation, and firm growth (as in Hsieh and Klenow (2012), Da-Rocha, Tavares, and Restuccia (2014) and Cole, Greenwood, and Sanchez (2012)) and ultimately, aggregate growth (as in Akcigit, Alp, and Peters (2014), Peters (2011)).

Peters (2011) links static misallocation (through markup variation) with innovation incentives and growth. Akcigit, Alp, and Peters (2014) provides a theory of firm dynamics in developing countries based on contracting frictions that prevent managerial delegation in weak institutional environments, i.e. poor economies. Both Akcigit, Alp, and Peters (2014) and Peters (2011) follow the Klette and Kortum (2004) tradition in that firms innovate by deciding the frequency of upgrades. Distinctively, we fix the frequency of innovation episodes, but we allow firms to choose the intensity in the improvement. This is important because in an environment where firms only decide the frequency of upgrades, steep employment age profiles are always coupled with faster growing economies. As mentioned before, this fact is at odds with the data. We are able to accommodate slow growing economies and steep employment age profiles by decoupling success rates from return uncertainty.

Hsieh and Klenow (2012) analyze employment age profiles in India, Mexico and the US to argue that about $25 \%$ of the differences in aggregate TFP can be accounted by disparities in firm employment growth. When analyzing a general equilibrium model 
with endogenous productivity growth (as in Atkeson and Burstein (2010)), they adjust returns to innovation in each country by the revenue distortions uncovered from the mexican and indian data. Because employment profiles are flatter in these countries, implied tax distortions are higher for firms with higher productivity. Higher tax distortions for more productive firms, induce the model to predict flatter employment profiles. In this paper we take an alternative path. We argue that it is not the actual return that is lower for more productive larger firms, but the expected return, once adjusted by uncertainty. Whereas the same technologies might available in developed and developing countries, their expected returns are different. Furthermore, our result holds even assuming that uncertainty on returns is the same irrespective of firm size. Slower growth as the firm ages is not generated by productivity dependent penalties on innovation, but through a general equilibrium effect via endogenous selection, i.e. entry and exit.

Another piece of research that argues that while the same technologies might be available across countries, features of the environment in which firms operate can generate disparities in the technological ladders that firms adopt, is Cole, Greenwood, and Sanchez (2012). The authors assert that poorer financial markets can explain the disparities in total factor productivity and employment size distribution. Contracting problems are undoubtedly a relevant mechanism that explains flatter productivity profiles. Akcigit, Alp, and Peters (2014), analyze contracting frictions that affect the ability of entrepreneurs to delegate managerial activities. Such friction can explain why it is optimal in certain economies for firms to remain small through time. Improvements in the contractual environment improve the net benefit of delegation and induces firm growth. In our paper, we vary the probability of obtaining returns to any given investment, conditional on the investment taken place. The presence of contracting frictions is definitely an important mechanism that would affect what we call the probability of success. There are other sources that our framework can also accommodate. For example, the existence of "social capital" as documented in Knack and 
Keefer (1997), and trust relationships, documented in Bloom and Sadun (2012); or the degree of technology experimentation, which has been shown key to predict innovation (Thomke (2003)). By keeping this reduced form approach, we gain model tractability and intuition as of the link between these alternative sources of uncertainty, the industry dynamics and growth. Certainly, the mechanism underlying firm uncertainty need to be further understood.

There is also a growing literature that studies firm growth in general equilibrium and its impact on aggregate allocation. Atkeson and Burstein (2010) study an open economy with endogenous growth. They focus on the impact of trade barriers on the equilibrium entry and exit rates and incentives to innovate. In their economy, firms choose the probability of realizing an improvement in productivity of a given size. Distinctively, our model takes the probability of success as a fundamental of the economy, and lets firms choose their path of productivity improvement. This is important because it allows us to fully characterize the equilibrium firm size distribution, which is key in validating our results.

Our paper is also related to the literature studying growth as the outcome of technological investment of heterogeneous firms operating in the market. Luttmer (2010) provides conditions such that the thick tail in the firm size distribution widely documented in the data can arise as the outcome of firms' growth under uncertainty. In our paper, we assume an initial distribution of productivity that has such a tail and hence we are silent about the process that originates it. This is also a feature of Perla and Tonetti (2014) that study diffusion of technology and growth in an economy with heterogeneous firms. Two features differentiate our model from theirs. First, when firms decide to invest in searching new technologies, the technology that is assigned to them is purely exogenous. In our model, firms decide on the optimal level of technology that they would like to run. Second, in Perla and Tonetti (2014) growth is lead by the firms at the bottom of the size distribution, who find it profitable to search better technologies. Bigger and more productive firms do not innovate. In our model, 
growth is lead by successful and fast growing firms. Small firms in the market invest in technology and only if successful, they turn large. If unsuccessful, they may opt out of innovation. The smallest firms in the market survive for a finite number of periods, and then endogenously exit.

The rest of the paper is organized as follows. Section 2 gathers empirical evidence across countries. Section 3 presents a stylized model that is consistent with those facts. Section 4 gives the analytical results from the model. Section 5 gives a quantitative assessment of the model. Section 6 introduces an extension of our benchmark model by allowing for additional idiosyncratic shocks affecting the size of firm growth. Section 7 concludes.

\section{Evidence}

We use the standardized dataset for 2006-2011 of the World Bank Enterprise Surveys (ES). We use data for 137 countries and for each country we pick the most recent survey available. When we split countries into income groups, we use the World Bank income classification thresholds defined for 2008 to form the groups of High Income, HighMiddle Income, Low-Middle Income and Low Income countries. ${ }^{4}$ Income classification is based on gross national income per capita, which we also get from the World Bank. To split the countries into growth groups, we take the following thresholds: economies with negative average annual growth (2000-2012); 0 to $1.5 \% ; 1.5 \%$ to $4 \% ; 4 \%$ to $8 \%$ and more than $8 \%$.

Since we argue that firms' probability of success has implications on income levels, we first analyze sales and employment information in the ES dataset. In particular, our objective is to show how the frequency of firms with positive growth in labor productivity relates with output growth and income per capita across countries. The fraction of firms that have positive productivity growth does not correspond to the

\footnotetext{
${ }^{4}$ Average year across the country specific surveys that we have is 2008.4.
} 
probability of success in attempts to increase productivity, a variable that we do not observe in the data. However, we expect such fraction to be increasing in the probability of success. High probability of success would increase the frequency of episodes with productivity growth for a given set of firms operating in the market. Selection may induce firms to exit more often, but if anything those would be relatively unsuccessful firms. Hence, we expect the fraction of firms with productivity growth to be increasing in the probability of success.

To compute growth in labor productivity, we compute the ratio of sales per worker in the survey year, and three years before the survey. Figure 1 shows this relationship between the fraction of firms with positive sales-per-worker growth within the last two years and average annual GDP growth as described before. The correlation coefficient is 0.23 in spite of the large variation across countries. Figure 2 shows the relationship between the fraction of firms with positive sales-per-worker growth and income per capita in the sample year. This correlation is still positive but weaker than the one for GDP growth (0.07).

In the appendix we consider measures of sales-per-worker growth adjusted by annual GDP growth of the economy. In other words, firm growth in excess to average growth of the economy for that particular time frame. The correlation between GDP growth and the fraction of firms with productivity increases is 0.4 , even higher than for the adjusted measure.

This initial set of evidence suggests that there is a link between probability of success and GDP growth.

Next we turn to the relationship between the distribution of employment among firms and income per capita. For this, we construct the Pareto tails for each country following Axtell (2001). We are interested in the tail of the distribution rather than other summary statistics for two reasons. First, the largest firms in each country contribute disproportionately more to value added, and explain most of the changes in aggregate output (as in Carvalho and Gabaix (2013)). Second, the survey considers 
Figure 1: Fraction of firms with productivity growth and GDP growth

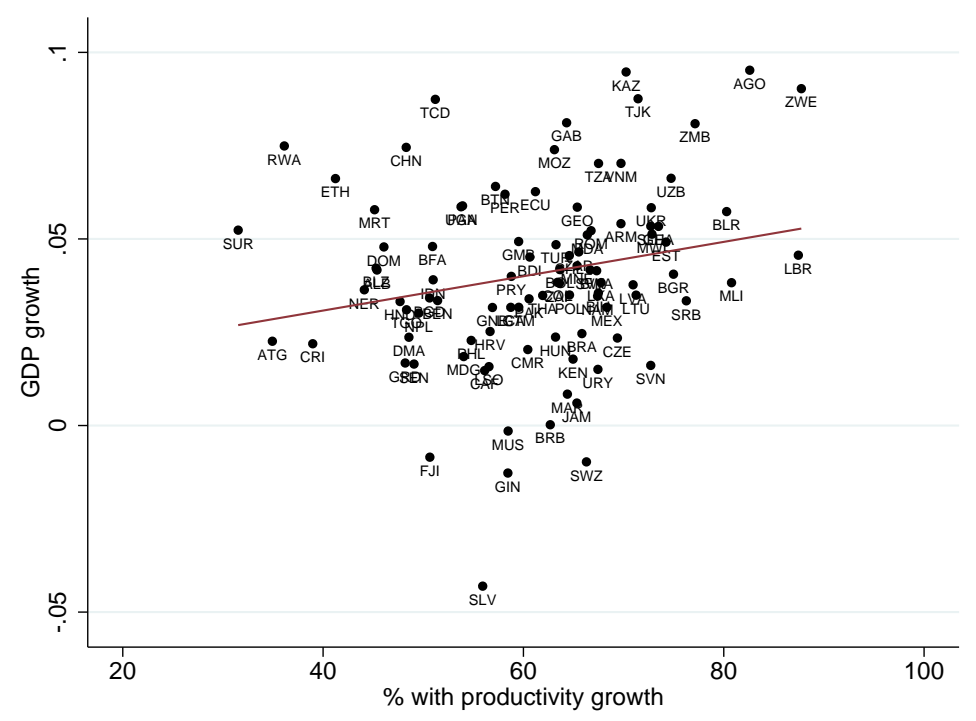

formal firms for most countries. In the presence of informal firms, the nature of this data could bias the analysis of the relationship between the employment size distribution and GDP growth. Our results are robust to analyzing firms that are larger than 5 employees. ${ }^{5}$

Our estimates for the tails of the distribution go from 1 to 4. A higher parameter indicates a thinner tail, i.e. there are relatively few large establishments employmentwise. Figure 3 shows that the thickness of the tail in the employment size distribution lacks systematic relationship with GDP growth (with correlation 0.01). Figure 4 shows a slight negative correlation (-0.18) between the thickness of the tail and income per

\footnotetext{
${ }^{5}$ In order to get the Pareto tail indices, we first form 15 employment categories, where category $i$ corresponds to $3^{i-1}$ integers. (The first category includes firms with 1 employee, the second category includes firms with 2 to 4 employees, so on.) For each country, we drop the categories that have less frequency that the one with higher order. Then we regress the logarithm of the number of firms on the logarithm of the median point of each category. The coefficient of the latter is the tail index for that economy. Pareto distribution captures the allocation of employment in these tails very well. Specifically, the average R-squared statistic in the regressions that we run to get the tail indices is 0.977 .
} 
Figure 2: Fraction of firms with productivity growth and GNI per capita

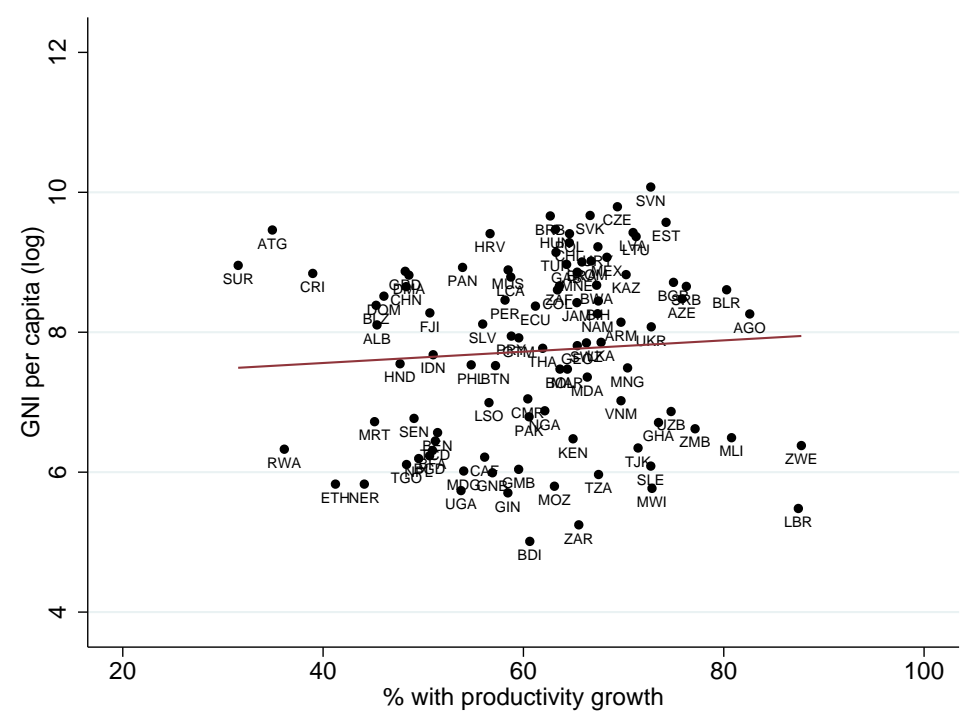

capita. In other words, richer countries have a larger proportion of high employment establishments. However, such proportion is not systematically related to the pace of growth in the economy. More importantly, it also lacks systematic relationship with the fraction of firms with positive productivity growth (with correlation -0.01).

Next, we analyze firm employment growth and its relationship to aggregate output growth and output per capita. To construct employment age profiles we use the cross sectional dimension of out dataset. To make profiles comparable across income and growth groups we normalize to 1 the predicted employment at age 5. Predicted employment is computed by fitting a quadratic polynomial of age on employment for each particular group. Figure 5 shows flatter profiles in fast growing economies, and steeper ones in slow growing economies. If instead we plot employment-age profiles by income group, we observe that on average they are steeper in richer economies. However, in our sample, these relationship is non-monotonous, as the poorest economies have steeper profiles that those observed in "Low-Middle Income" economies (See Figure 6).

The last piece of data that is important to complete our analysis is that of firm 
Figure 3: Tail indices and GDP growth

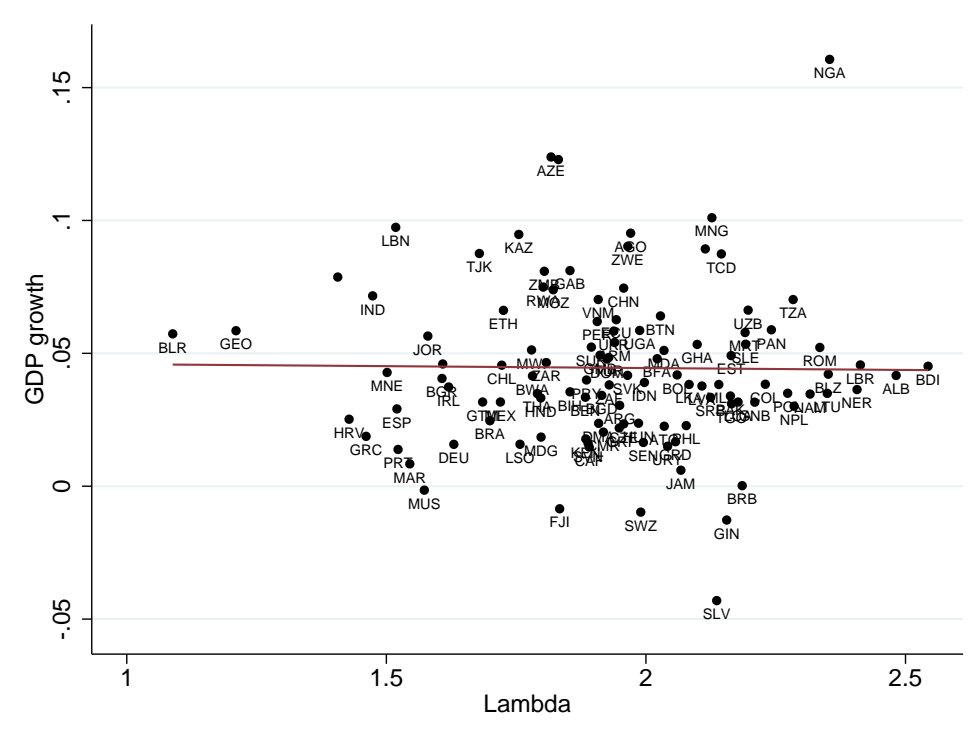

selection. The nature of the ES data does not allow us to track patterns of entry and exit of firms. However, Bartelsman, Haltiwanger, and Scarpetta (2009) have documented extensively the process of firm selection, patterns of entry and exit and growth for 24 economies. While this is a smaller set than the one available at the ES, it yields useful information as of the disparities in firm dynamics across countries at different stages of development. ${ }^{6}$ The fact of particular interest for us is the higher turnover for countries with relatively higher firm growth. To sum up, we would like to highlight three of the empirical facts in this section. First, there is a positive correlation between frequency of productivity growth at the firm-level and income per capita. Second, the tail of employment size distribution is not correlated with aggregate growth. Third, employment age profiles are steeper in slow growing economies.

\footnotetext{
${ }^{6}$ For emerging economies in particular, there is widespread evidence that the pattern of firm dynamics is key in explaining the success with which these economies opened to markets. (Eslava, Haltiwanger, Kugler, and Kugler (2004), Roberts and Tybout (1997))
} 
Figure 4: Tail indices and income per capita

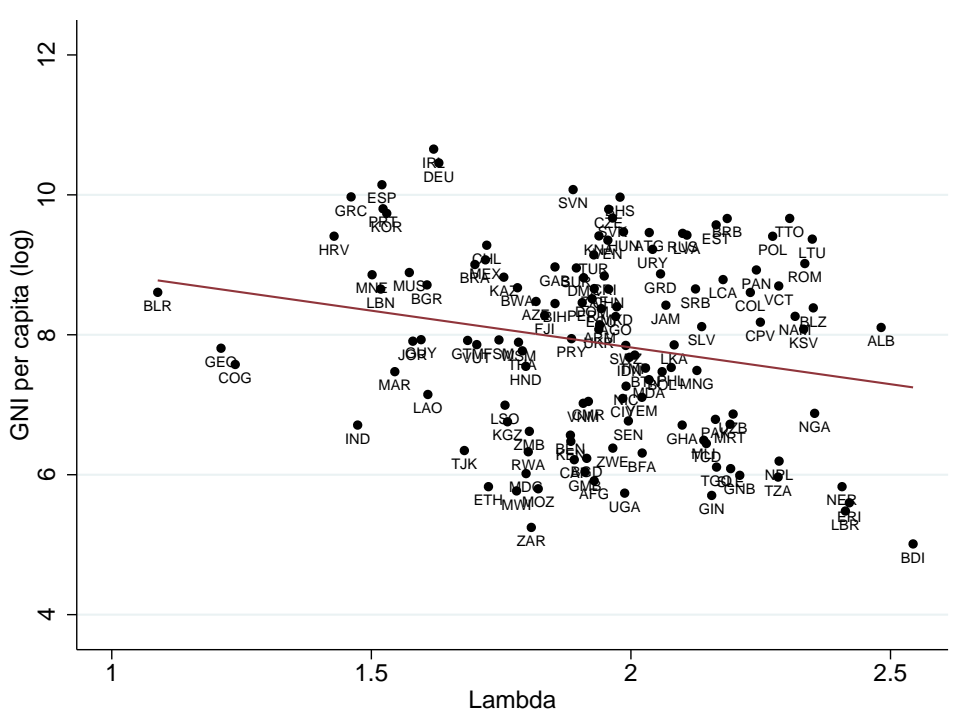

Figure 5: Employment-Age profiles by growth group

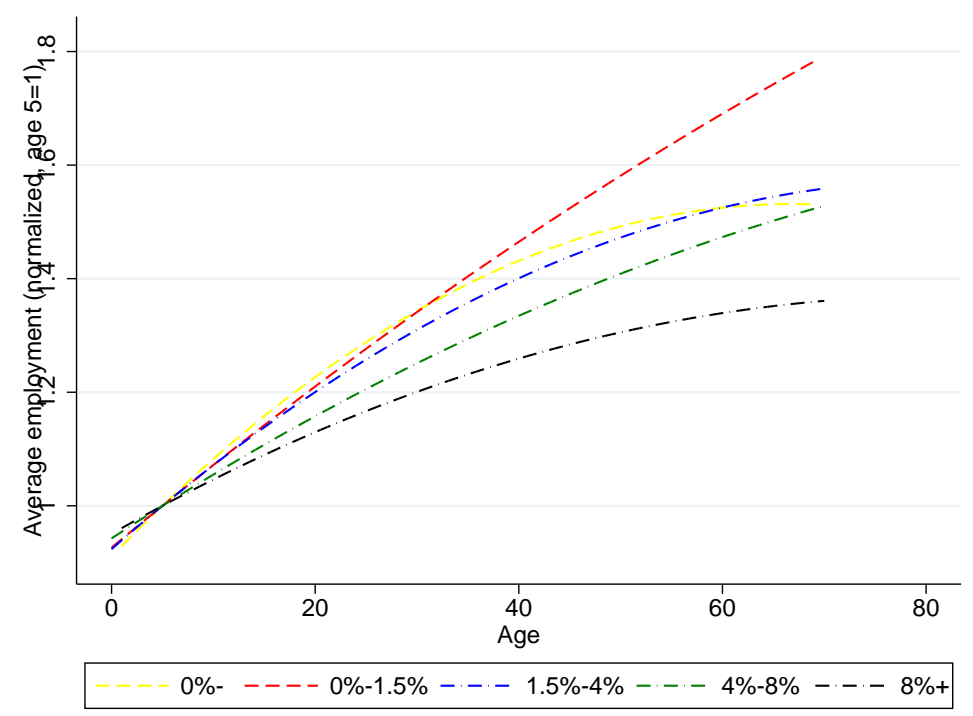


Figure 6: Employment-Age profiles by income group

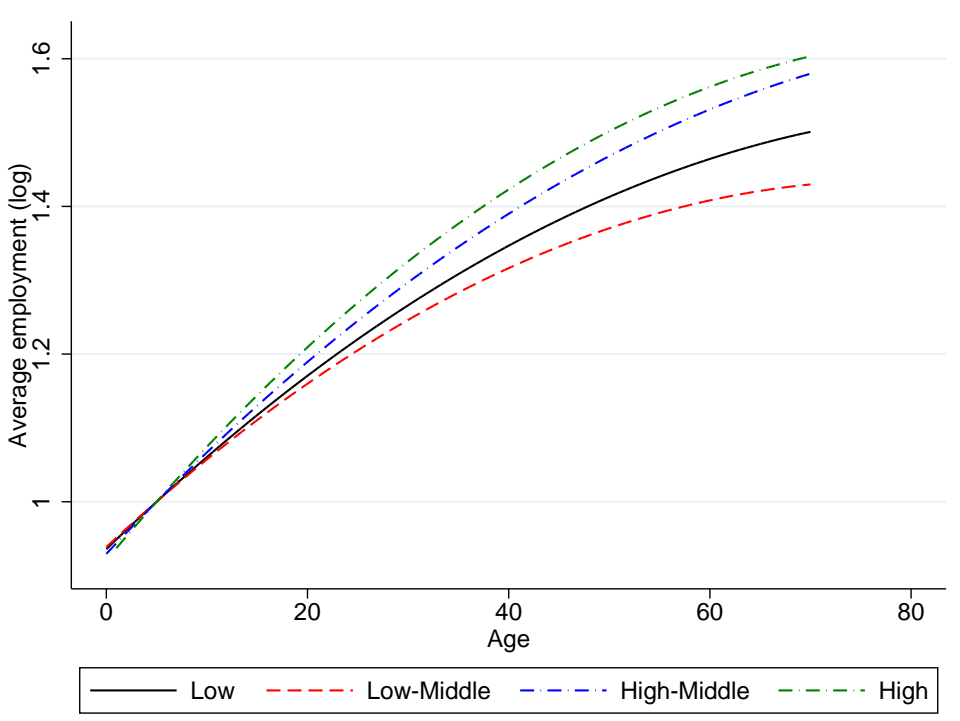




\section{Model}

In the economy, there is an infinitely lived representative consumer that derives utility from consumption of a final good $y$.

There is a continuum of heterogeneous firms. Each firm operates a technology that uses labor $l$ to produce a homogeneous good $y$. The technology displays decreasing returns in labor, and has a Hicks neutral productivity shifter $z^{\zeta}$. Hence, after paying workers their wage rate $w$ the firm bears positive profits that are paid as dividends to the representative consumer in the economy.

There are two alternative types of firms. The non-innovative ones, whose productivity level $z$ is constant in time; and the innovative ones, whose productivity can change via investment. The technology for improvements in productivity is such that whenever a firm with productivity $z$ undertakes an investment $\phi$ in period t, the productivity in period $t+d t$ is $z^{\prime}=e^{\phi d t} z$ with probability $q$ and $z$ otherwise. The probability of success in investment returns is the same for all firms operating in the economy. Investment is costly, as characterized by $C(\phi, z, w)=c \frac{e^{\phi \tau d t} z^{\eta}}{w^{\frac{\theta}{1-\theta}}}$.

There is an inelastic supply of labor equal to 1. A firm has an overhead cost of labor of $f$ workers irrespective of whether the firm is innovative or non-innovative.

Both types of firms can decide to exit the market at no cost at any point in time. An innovative firm has the additional option of liquidation. Every period an innovative firm is matched to a potential entrant to the non-innovative sector, which makes a take-it-orleave-it offer to obtain the production technology of the former. If the transaction goes through, the buyer starts operating in the non-innovative sector and the seller leaves the market. Notice that if the innovative firm exits without liquidating, it gets a scrap value of zero; hence liquidation is preferable as long as the non-innovative projects are valuable. Finally, firms are subject to exogenous exit shocks arriving at rate $\delta$ before production and investment takes place.

We now characterize the optimization of each of the agents in the economy. We can 
solve the problem of the firms in two parts. First we solve for the allocation of labor given the distribution of productivity in the market. Then we solve for the dynamic decisions of the firm, which include technology investment.

\subsection{Firms' problem (static)}

The static problem of a firm is:

$$
\Pi(z, w)=\max _{l} z^{\zeta} l^{\theta}-w l
$$

where $\theta$ is the share of labor in production.

Then optimality implies

$$
l=\left(\frac{\theta z^{\zeta}}{w}\right)^{\frac{1}{1-\theta}}
$$

For notational convenience, define $\eta \equiv \frac{\zeta}{1-\theta}$.

The total labor supply that can be used for productive purposes is equal to $1-M f$, if there are $M$ firms operating in the market $\left(M=M_{I}+M_{N}\right.$ where $M_{I}$ is the measure of innovative firms and $M_{N}$ that of non-innovative firms). We can solve for the cost of labor

$$
w=\frac{\theta}{(1-f M)^{1-\theta}}\left(\int z^{\eta} d v^{I}(z)+\int z^{\eta} d v^{N}(z)\right)^{1-\theta}
$$

where $v^{I}\left(v^{N}\right)$ is the equilibrium distribution of productivities for innovative (noninnovative) projects. By definition, $M_{j}=\int d v^{j}(z)$ for $j=I, N$.

Using the equilibrium cost of labor we can characterize profits, labor demand and output for an arbitrary firm with productivity $z$ :

$$
\Pi(z, w)=(1-\theta) \theta^{\frac{\theta}{1-\theta}} \frac{z^{\eta}}{w^{\frac{\theta}{1-\theta}}}
$$




$$
\begin{aligned}
& l(z, w)=\theta^{\frac{1}{1-\theta}} \frac{z^{\eta}}{w^{\frac{1}{1-\theta}}} \\
& y(z, w)=\theta^{\frac{\theta}{1-\theta}} \frac{z^{\eta}}{w^{\frac{\theta}{1-\theta}}}
\end{aligned}
$$

\subsection{Firms' problem (dynamic)}

\subsubsection{Non-innovative firms}

Let $V_{N}(z, w)$ be the value of a firm operating in the non-innovative sector, when the cost of labor is $w$ and its productivity is $z$. The value of a firm operating in sector $\mathrm{N}$ satisfies:

$$
V_{N}(z, w)=(1-\theta) \theta^{\frac{\theta}{1-\theta}} \frac{z^{\eta}}{w^{\frac{\theta}{1-\theta}}}-f w+e^{-(r+\delta) d t} \max \left\{0, V_{N}\left(z, w^{\prime}\right)\right\}
$$

In an abuse of notation we are denoting by $w^{\prime}$ the wage rate at time $t+d t$.

The value of the firm is negative if and only if the flow value in that period is negative. i.e.

$$
V_{N}(z, w) \leq 0 \Leftrightarrow(1-\theta) \theta^{\frac{\theta}{1-\theta}} \frac{z^{\eta}}{w^{\frac{\theta}{1-\theta}}}-f w \leq 0 .
$$

Notice that the only decision for this firm is when to exit the market, conditional on survival.

\subsubsection{Innovative firms}

An innovative firm has the same static revenues as a non-innovative firm, but is different in two important aspects. First, it can engage in risky investment in productivity that generates successful innovation only with probability $q$. Second, at any period it can sell its technology to an entrant into the non-innovative sector. We can write the value of an innovative firm as: 


$$
\begin{gathered}
V_{I}(z, w)=\max _{\phi \geq 1}(1-\theta) \theta^{\frac{\theta}{1-\theta}} \frac{z^{\eta}}{w^{\frac{\theta}{1-\theta}}}-c \frac{e^{\phi \tau d t} z^{\eta}}{w^{\frac{\theta}{1-\theta}}}-f w \\
\left.+e^{-(\delta+r) d t}\left[q \max \left\{V_{I}\left(e^{\phi d t} z, w^{\prime}\right), P\left(z, w^{\prime}\right)\right\}+(1-q) \max \left\{V_{I}\left(z, w^{\prime}\right), P\left(z, w^{\prime}\right)\right)\right\}\right]
\end{gathered}
$$

Here we assume that if a firm sells the technology in a period, this nullifies any improvement in the technology that was made on productivity in the last period. This is analogous to assuming that the liquidation of the firm takes place before the firm knows whether their technology investment was successful or not.

If the firm would like to liquidate, at the beginning of a period it meets one potential entrant that will use its technology $z$ to operate a non-innovative firm. The latter makes a take-it-or-leave-it offer $P(z, w)$ to the former to buy the entire technology.

It is optimal for the entrant to offer:

$$
P(z, w)=\min \left\{V_{I}(z, w), V_{N}(z, w)\right\}
$$

and the innovative firm accepts if and only if

$$
P(z, w) \geq V_{I}(z, w)
$$

In other words, the transaction occurs when its surplus is positive. With this pricing scheme, we have $\max \left\{V_{I}\left(e^{\phi d t} z, w^{\prime}\right), P\left(z, w^{\prime}\right)\right\}=V_{I}\left(e^{\phi d t} z, w^{\prime}\right)$ and $\max \left\{V_{I}\left(z, w^{\prime}\right), P\left(z, w^{\prime}\right)\right\}=$ $V_{I}\left(z, w^{\prime}\right)$. Hence, an innovative firm makes its technology investment decisions as if it was operating an infinitely lived firm.

Proposition 1 Along the BGP, for every innovative firm there exists a time $\hat{t}<\infty$ that it sells its technology to an entrant non-innovative firm.

As shown in the proof in the Appendix, for the technology to be changing hands between an $I$-firm and an $N$-firm, we need (i) the surplus in the transaction to increase in unsuccessful innovations. Hence, unlucky firms eventually would like to liquidate. 
We also need that (ii) for a sufficiently large $z$ relative to $w$ the surplus is negative (a very productive firm does not find profitable to liquidate), and (iii) for a sufficiently small $z$ relative to $w$ the surplus is positive. If this is true there is a finite point in time such that an innovative firm is traded. Hence, the population of both types of firms is non-degenerate.

Under this trading scheme, the value of an innovative firm is:

$$
\begin{gathered}
V_{I}(z, w)=\max _{\phi \geq 1}(1-\theta) \theta^{\frac{\theta}{1-\theta}} \frac{z^{\eta}}{w^{\frac{\theta}{1-\theta}}}-c \frac{e^{\phi \tau d t} z^{\eta}}{w^{\frac{\theta}{1-\theta}}}-f w \\
+e^{-(\delta+r) d t}\left[q V_{I}\left(e^{\phi d t} z, w^{\prime}\right)+(1-q) V_{I}\left(z, w^{\prime}\right)\right]
\end{gathered}
$$

\section{Solving the model}

\subsection{Balanced Growth Path}

Definition 1 A Balanced Growth Path (BGP) in this economy is a sequence of aggregate output, a measure of aggregate productivity and wages that grow at a constant rate. Additionally, we require the existence of an invariant distribution of firm productivities except possibly for a time trend.

To characterize the BGP we guess there exists one. Under this assumption we solve for the optimal policies of the firms and show the existence of an invariant distribution. Once we solve for the equilibrium distribution and allocation of firms across innovative and non-innovative projects, we compute the equilibrium growth rate for aggregate output and wages. Both are constant, which confirms the existence of the BGP.

Aggregate output in the economy can be characterized by

$$
Y(w)=\frac{w}{\theta}
$$

hence along a BGP output and wages have to grow at the same rate. i.e. 


$$
\left[Y_{t}, w_{t}\right]=[Y, w] e^{\gamma t}
$$

for some $\gamma \geq 0$. Using the parametrized growth rate for wages we can go back to the problem of the firms.

\subsubsection{Non-innovative firms}

Define the function for the number of periods left in the market as:

$$
T(z, w) \equiv \min \left\{t:(1-\theta) \theta^{\frac{\theta}{1-\theta}} z^{\eta} \leq f w^{\frac{1}{1-\theta}} e^{\frac{\gamma}{1-\theta} t}\right\}
$$

We will simply denote this as $T$.

Proposition 2 Along the BGP, the value of a firm at the beginning of a period can be characterized by

$$
W_{N T}(z, w)=\max \left\{0, V_{N}(z, w)\right\}=B_{N T} \frac{z^{\eta}}{w^{\frac{\theta}{1-\theta}}}-D_{N T} w
$$

where for any $T \geq 1$ :

$$
\begin{gathered}
B_{N T}=(1-\theta) \theta^{\frac{\theta}{1-\theta}} \exp \left(-\left(\delta+r-\gamma \frac{\theta}{1-\theta}\right) T\right) \\
D_{N T}=f \exp (-(\delta+r-\gamma) T)
\end{gathered}
$$

and for $T=0$ both coefficients are 0 .

If the functions $B_{N T}$ and $D_{N T}$ are as characterized above, the non-innovative firm exits the market in $T$ periods and $W_{N T}(z, w)$ satisfies the Bellman equation, which assures optimality. 


\subsubsection{Innovative firms}

We guess that the value of an innovative firm is

$$
V_{I}(z, w)=B_{I} \frac{z^{\eta}}{w^{\frac{\theta}{1-\theta}}}-D_{I} w
$$

Replacing it in the Euler equation yields

$$
\begin{gathered}
B_{I} \frac{z^{\eta}}{w^{\frac{\theta}{1-\theta}}}-D_{I} w=\max _{\phi \geq 1}(1-\theta) \theta^{\frac{\theta}{1-\theta}} \frac{z^{\eta}}{w^{\frac{\theta}{1-\theta}}}-c \frac{e^{\phi \tau d t} z^{\eta}}{w^{\frac{\theta}{1-\theta}}}-f w \\
+e^{-(\delta+r) d t}\left[q\left(B_{I} \frac{e^{\phi \eta d t} z^{\eta}}{w^{\frac{\theta}{1-\theta}}}-D_{I} w^{\prime}\right)+(1-q)\left(B_{I} \frac{z^{\eta}}{w^{\frac{\theta}{1-\theta}}}-D_{I} w^{\prime}\right)\right]
\end{gathered}
$$

Then solving the FOC with respect to $\phi$ yields

$$
z^{\prime}=e^{\phi d t} z=\left(\frac{e^{\widehat{\beta} d t} q B_{I} \eta}{c \tau}\right)^{\frac{1}{\tau-\eta}} z
$$

where $\widehat{\beta}=-\left(\delta+r-\gamma \frac{\theta}{1-\theta}\right)$

Substituting back

$$
B_{I} \eta=\eta(1-\theta) \theta^{\frac{\theta}{1-\theta}}-c(\eta-\tau)\left(\frac{e^{\widehat{\beta} d t} q B_{I} \eta}{c \tau}\right)^{\frac{\tau}{\tau-\eta}}+e^{\widehat{\beta} d t}(1-q) B_{I} \eta
$$

gives the first equation relating $\phi$ and $\gamma$ through $B_{I}$.

It is important to point out that we have assumed an interior solution. In particular, we assumed that the firm with optimal investment rule as defined above remains in the market after a successful innovation. We will later show that this actually holds in equilibrium. 


\subsection{Distribution dynamics}

Define the threshold level of productivity such that a non-innovative firm exits in exactly $t$ periods, $Z(t, w)$ as

$$
Z(t, w)=\left(\frac{f w^{\frac{1}{1-\theta}} e^{\frac{\gamma}{1-\theta}} t}{(1-\theta) \theta^{\frac{\theta}{1-\theta}}}\right)^{\frac{1}{\eta}}
$$

The lower bound in productivity for firms operating in the market is $\tilde{z}$, which solves:

$$
(1-\theta) \theta^{\frac{\theta}{1-\theta}} \tilde{z}^{\eta}=f w^{\frac{1}{1-\theta}}
$$

In particular, the lower bound for the productivity of firms that exit immediately is $\tilde{z}=Z(0, w)$. If aggregate output grows at rate gamma along the BGP, the lower bound in productivities for incumbent firms evolves as

$$
\tilde{z}^{\prime}=\tilde{z} e^{\mu d t}
$$

where $\mu \equiv \frac{\gamma}{\eta(1-\theta)}$.

Finally, the threshold of productivity for firms that exit in $\hat{t}$ periods is

$$
\hat{z}=Z(\hat{t}, w)
$$

which also grows at rate $\mu$.

Proposition 3 If the initial distribution of productivities in the market is Pareto with shape parameter $\lambda$, and entrants in the innovative sector draw their productivity from the incumbent distribution

1. the growth rate of the threshold levels, $\mu$, is the same as the investment rate, $\phi$. 
2. the equilibrium distribution of productivities across innovative firms, $F_{I}(z)$, is also Pareto with shape parameter $\lambda$.

This result implies that

$$
\phi=\frac{\gamma}{\eta(1-\theta)}
$$

which is the second equation relating $\gamma$ and $\phi$. It also shows that there is an invariant distribution of firms in the market.

It remains to be shown that the relative population of innovative and non-innovative projects in the market is constant along the BGP. Let $\alpha$ be the proportion of firms in the market in the innovative sector, i.e. $\alpha \equiv \frac{M_{I}}{M}$.

Proposition 4 The share of innovative firms in the market is constant along the BGP and solves

$$
(1-\alpha)=\alpha(1-q)\left[1-e^{-\mu \lambda}\right] e(-\delta T)
$$

Before moving on to the quantitative section of the paper, it is important to characterize the measure of total factor productivity in the economy. Aggregate output can be written as

$$
Y_{t}=T F P_{t} \cdot 1
$$

as the aggregate endowment of labor is constant equal to one. Using the equilibrium wages in the economy, the optimal liquidation and exit strategies, we can characterize TFP as a function of the equilibrium allocation of firms across technologies and productivities.

$$
T F P=(1-f M)^{\theta}\left(\int_{\widehat{z}}^{\infty} z^{\eta} d F_{I}(z)+\int_{0}^{\hat{t}-d t}\left(\int_{\widetilde{z} e^{\mu s}}^{\widetilde{z} e^{\mu(s+d t)}} z^{\eta} d F_{N}(z, s)\right) d s\right)^{1-\theta}
$$

where $F_{N}(z, s)$ is the distribution of non-innovative firms, with $s$ periods left in the market. 
As it is usual in models with a non-degenerate distribution of firms in the market, TFP depends on the number of operating firms and the degree of curvature in the profit function of firms in the market, $\theta$. Particular to this model is the dependence of TFP on the allocation of firms between the innovative and non-innovative sector, as well as on the time span over which non-innovative firms survive in the market, $\hat{t}$. Faster growing economies will have lower $\hat{t}$ so even if the share of firms innovating in the market is the same as that of a slower growth economy, TFP will be higher in the former.

\section{Quantitative exploration}

This section is split in two. First we show the general characteristics of the solution for an arbitrary parametrization of the economy. Then we calibrate the model to a benchmark high income economy and compare the predictions of the model for alternative slow growing economies.

Given the stylized characteristics of our model, we can use two equations to find the equilibrium growth rate in the economy, as well as the growth rates at the firm level.

$$
\begin{gathered}
\phi=\frac{\gamma}{\eta(1-\theta)} \\
\frac{c \tau e^{\phi(\tau-\eta) d t}}{e^{\widehat{\beta} d t}}=\eta(1-\theta) \theta^{\frac{\theta}{1-\theta}}-c(\eta-\tau) e^{\phi \tau d t}+\frac{1-q}{q} c \tau e^{\phi(\tau-\eta) d t}
\end{gathered}
$$

Notice that the first equation was derived while computing the existence of an invariant distribution, under the assumption of an initial Pareto distribution. At that point we showed that the equilibrium invariant distribution would be Pareto with lower bounds that grow at the investment rate of innovative firms in the market. The investment rate and growth rate of the economy are hence independent of the fraction of innovative firms (the parameter $\alpha$ ) as well as the measure of the firms operating in the market.

Solving for the growth rate of the economy and the investment rate of the firms, 
Figure 7: Equilibrium growth rate and firm investment rate

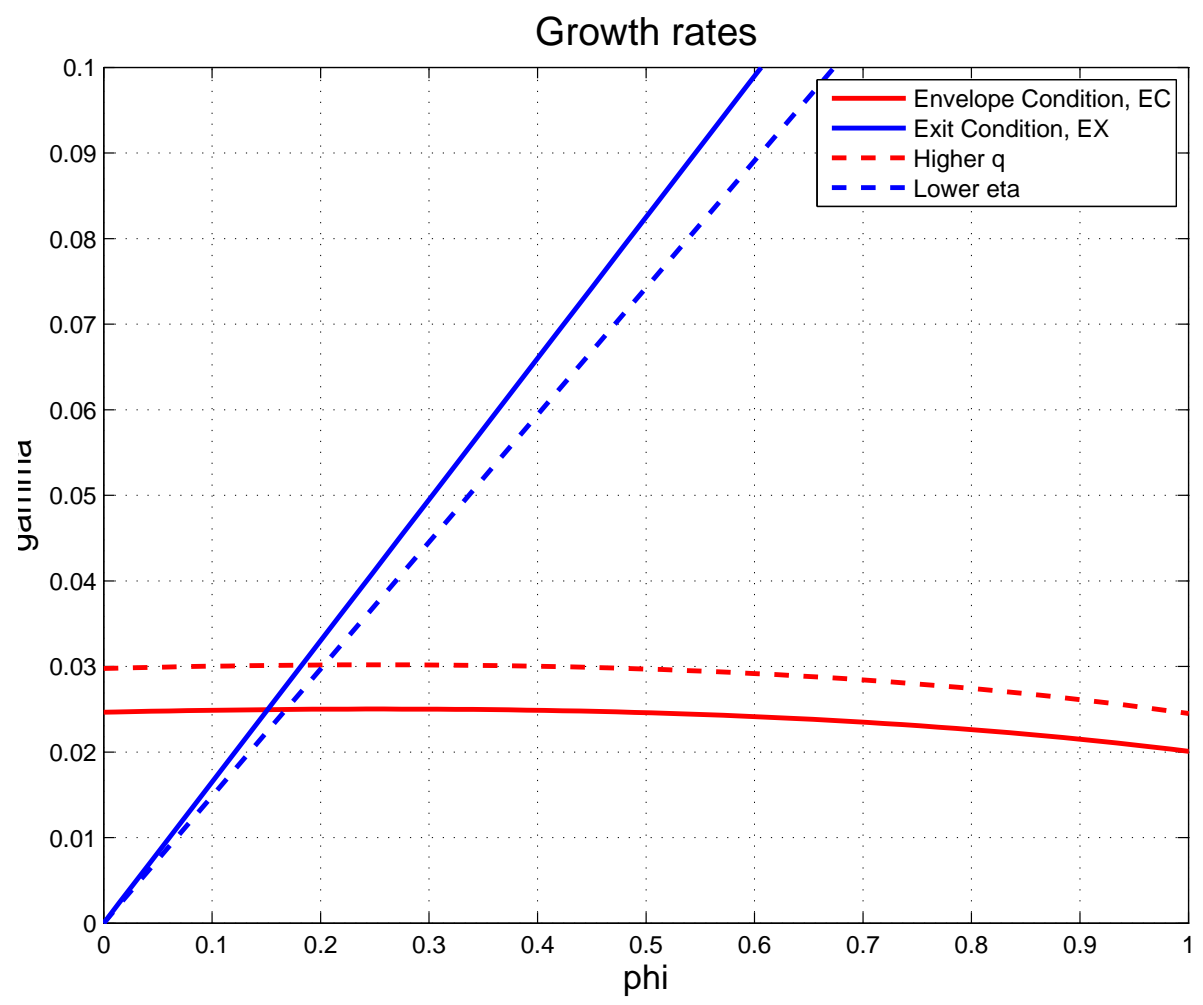

Figure 7 displays the equilibrium growth rates for an arbitrary parametrization of the model and alternative probabilities of success. As expected, we find that the higher the probability of success ( $q$ goes from 0.5 to 0.9 ), the higher is the investment rate of the firms in the economy, and the higher is aggregate growth. Given the curvature in the profit function, an increase and a decrease of the same magnitude in the probability of success does not have a symmetric impact on neither the investment rates nor on the growth rate of the economy. The curvature of the profit function as characterized by $\theta$ is key in explaining the asymmetry.

Changes in the probability with which firms realize returns to investment, $q$, also has an impact on the equilibrium number of firms operating in the market, as well as 
on the distribution of firms between the innovative and non-innovative sector. Figure 8 displays the equilibrium measure of firms operating in the market, and the share of non-innovative firms for an arbitrary parametrization (in red). When we lower the probability of success from 0.5 to 0.4 , the equilibrium measure of firms in the market increases. There is less competition for resources and equilibrium profits are higher, which induces entry to satisfy the free entry condition. The share of non-innovative firms in the market also increases, as firms in the innovative sector are more likely to observe a stream of bad draws in which they do not realize the returns to their technology investment. Notice that when the probability of success decreases, the share of noninnovative firms increases monotonically. However, the measure of firms in equilibrium may not rise. The optimal exit strategy depends on the equilibrium profits of the innovative firms in the market, which may rise or fall with smaller $q$ through general equilibrium effects. To put it simply, it depends on whether the equilibrium profits of the firms operating in the market decrease or not.

Now we calibrate the model to a benchmark high income economy. Important parameters that need to be identified in the data are the cost of investment, $c$, and the parameter $\eta$ that is related to the curvature in the profit function. To do it, we use equations 1 and 2 for given values of the aggregate growth rate of the economy, $\gamma$, growth rate of firms, $\phi$, and the probability of success, $q$. The first one is calibrated to the average GDP growth of the US economy in the last 50 years. Firm growth is computed to match the implied yearly employment growth of firms between ages 5 and 10 for high income economies. In the model there is exogenous and endogenous exit. Endogenous exit is explained by the firms at the bottom of the distribution. Hence, the exogenous rate is calibrated to match the exit rate in the left tail of the size distribution. According to the Business Dynamics Statistics by US Census, the average exit rate of establishments with more than 1000 employees (1978-2010) is 0.012; this is the rate that we use for $\delta$, the rate of exogenous exit in our economy. We calibrate the probability of success using data from the Panel Study of Entrepreneurial Dynamics (PSED). We 
Figure 8: Measure of firms and optimal allocation to innovation. Baseline $q=0.5$ (red), alternative $\mathrm{q}=0.4$ (black)

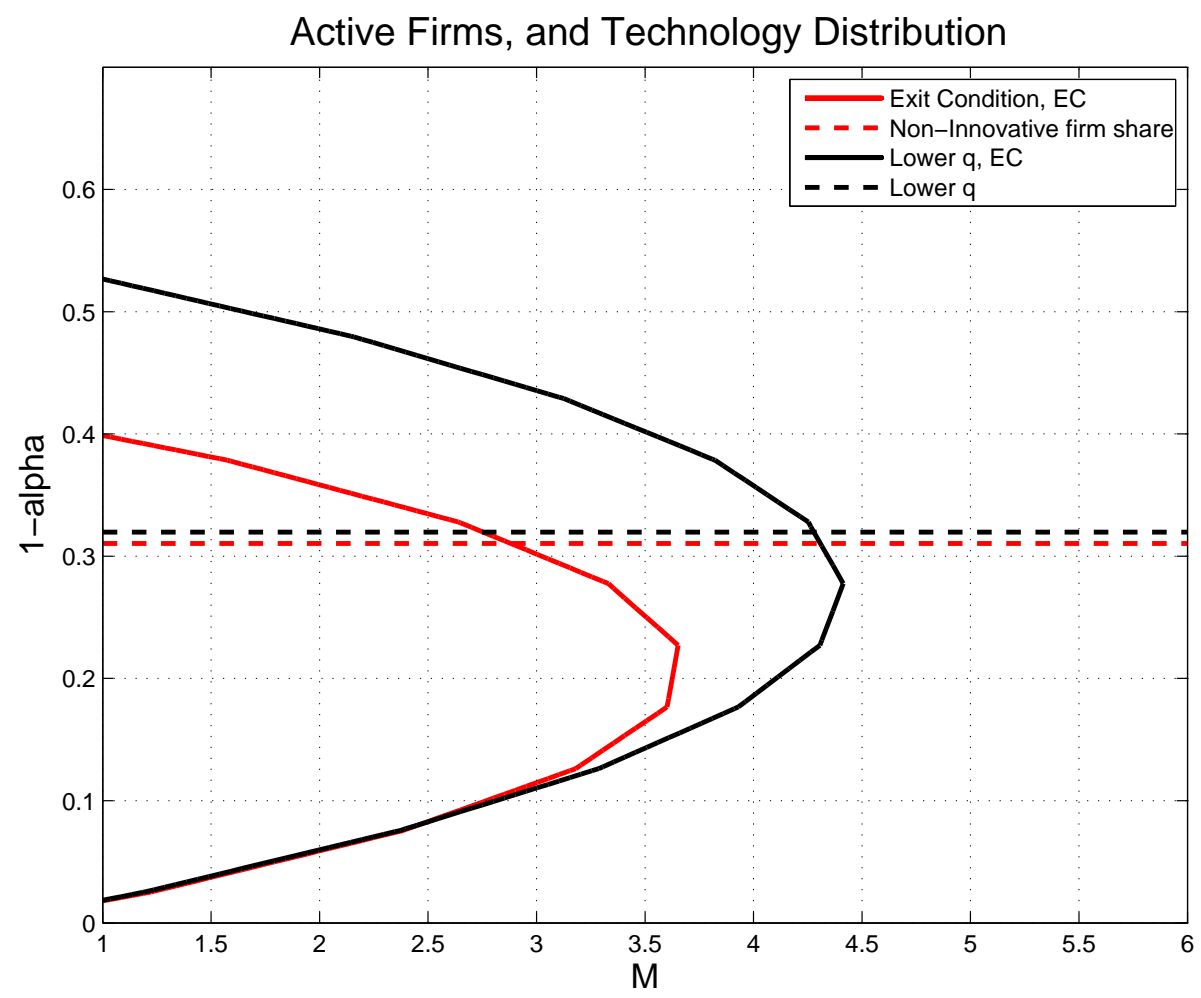

target the fraction of firms with positive growth in 4 years of operations, within the ones declaring that they would like to grow. In the data this rate is $97 \%$, which would yield a probability of success of $59 \%$ for one year. We also need to calibrate the non-linear term in the cost structure, $\tau$. We set it equal to 1.6 and later run sensitivity analysis on this parameter. This is also our strategy for the parameter $\theta$, which in the baseline economy is set to 0.85 . This value for the span of control corresponds to the baseline value in Atkeson and Kehoe (2005), when quantifying organizational capital for the US economy. The net interest rate in the market is set to $2 \%$ per year. Table 1 summarizes the parameters used in the benchmark model. ${ }^{7}$

\footnotetext{
${ }^{7}$ We will include sensitivity analysis for the overhead costs, $f$, in the later draft.
} 
Table 1: Benchmark calibration

\begin{tabular}{cccc}
\hline DESCRIPTION BENCHMARK & SYMBOL & VALUE & BASIS \\
\hline Aggregate Growth & $\gamma$ & $2 \%$ & GDP growth \\
Firm Growth & $\phi$ & $4.6 \%$ & WB Average Profile \\
Span of Control & $\theta$ & 0.85 & Atkeson and Kehoe (2005) \\
Probability of Success & $q$ & 0.59 & Expected Growth (PSED) \\
Investment Cost (non-linear component) & $\tau$ & 1.6 & See text. \\
Exogenous Exit Rate & $\delta$ & 0.012 & Exit rate for large firms (BDS) \\
Overhead Cost & $f$ & 0.1 & See text. \\
Interest Rate & $R$ & 0.02 & Risk-free rate \\
\hline \hline Investment Cost (linear component) & $c$ & 0.05 & Calibrated \\
Curvature Profit Function, given $\theta$ & $\eta$ & 2.9 & Calibrated \\
\hline
\end{tabular}

The model implies that for the solution to be well defined we need the Pareto tail parameter to exceed the curvature in the profit function: $\lambda>\eta$. When we estimate log-log regressions in employment shares by employment classes (see Section 2), we obtain a Pareto Tail for high income economies of 1.96, which is below our estimated $\eta$ parameter. We compute the baseline allocation for $\lambda=1.1 \eta$.

Given these parameters, we can compare the predictions in our benchmark economy with those in an economy with lower aggregate growth. We use $\gamma_{A C}<\gamma$ and compute the implied probability of success $q$ for $c$ and $\eta$ constant across economies.

For an economy that grows 0.05 percent less each year $\left(\gamma_{A C}=0.0195\right)$, we obtain that the average growth of firms if successful in growing drops by 0.1 percent to $\phi_{A C}=$ 0.045. The implied probability of success also drops by 1.25 percent from 0.59 to 0.5675 . Selection of firms in the market changes as well. A non-innovative firm remains in the market $1.03 \%$ longer than in the benchmark economy. The overall number of firms operating in the market increases $1.3 \%$ and the proportion of innovative firms in the market drops $1.6 \%$.

Next, we do comparative statics using alternative parametrizations for the bench- 
Table 2: Alternative economies

\begin{tabular}{cccc}
\hline & Baseline & Alt. ECONOMY & UpPeR-MidDlE \\
\hline$\gamma$ & $2 \%$ & $1.95 \%$ & $1.2 \%$ \\
$q$ & 0.59 & 0.5675 & 0.26 \\
$\phi$ & $4.6 \%$ & $4.5 \%$ & $2.8 \%$ \\
$M$ & 3.01 & 3.05 & 3.99 \\
$\alpha$ & 0.894 & 0.88 & 0.88 \\
$\widehat{t}$ & 2.4 & 2.46 & 2.16 \\
\hline
\end{tabular}

mark probability of success, $q$; for the non-linear term in the cost structure, $\tau$; and for the curvature in the profit function, $\theta$.

Figure 9 shows that the identified cost parameter $c$ is not very sensitive to the underlying choice of the probability of success. The implied probability of success in the alternative country $q_{A C}$ is roughly $1.5 \%$ lower than $q$ in the baseline economy. The picture is different when we look at the share of innovative firms in the market. The differences between the benchmark and the alternative economy drop as the probability of success increases. For relatively low and high probability of success, the share of innovative firms in the market is higher in the benchmark economy than in the alternative one. For medium values of the probability of success the share of innovative firms is higher in the slow growing economy. This disparity has to do with the impact on equilibrium profits of changes in the probability of success. When we have a slow growing economy, less competition for resources induces lower labor cost and higher equilibrium profits, which may induce more firms to innovate. The impact on profits is a general equilibrium one and depends on the incentives for entry and exit, and the new equilibrium measure of firms operating in the market. Consistently with Figure 7 the measure of firms can be lower or higher in the alternative economy than in the benchmark economy. When the underlying probability of success is high, it is possible that the economy with lower aggregate growth has more firms operating in the market in equilibrium. When the probability of success is relatively low, a slow growth economy 
Figure 9: Measure of firms and optimal allocation to innovation. Baseline $q=0.59$ (blue), alternative $\mathrm{q}=0.581$ (red)
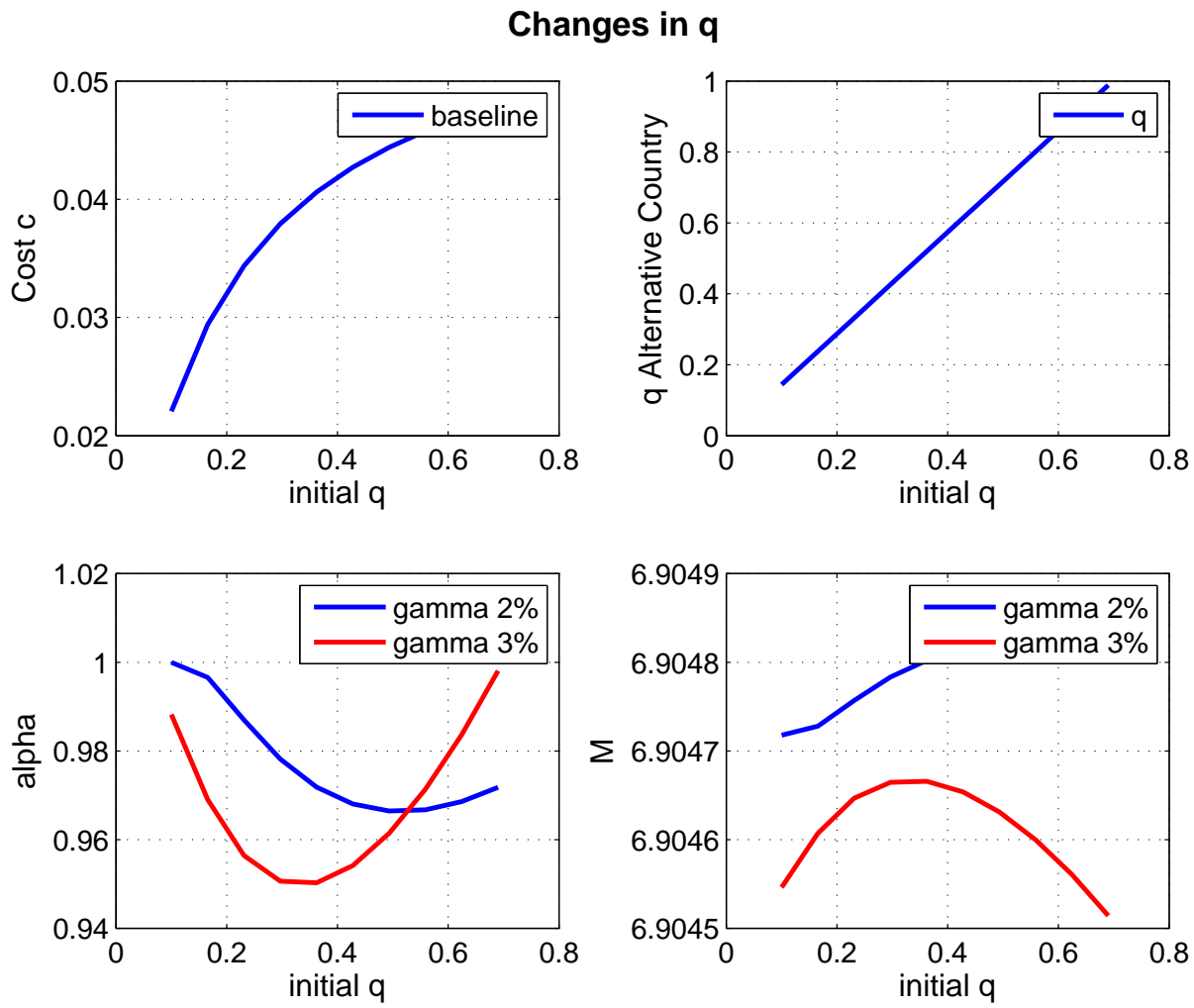

displays a lower measure of firms operating in equilibrium.

Figure 10 shows the response of the cost parameter to changes in the curvature of the cost function as measured by $\tau$. The linear component is decreasing in the curvature of the cost function. The probability of success in the alternative economy, is not very sensitive to changes in the curvature parameter, partly because the linear component of the cost structure does not vary much with $\tau$. In terms of the predicted measure of firms in either economy, as we increase $\tau$ the measure of firms in the slow growth economy becomes higher than the measure in the benchmark economy. For $\tau$ close to 2 the difference is three times as large as for $\tau$ equal 1.6. The predicted share of innovative firms in the market is lower in the slow growing economy for relatively low 
Figure 10: Comparative statics, changes in the curvature of the cost function
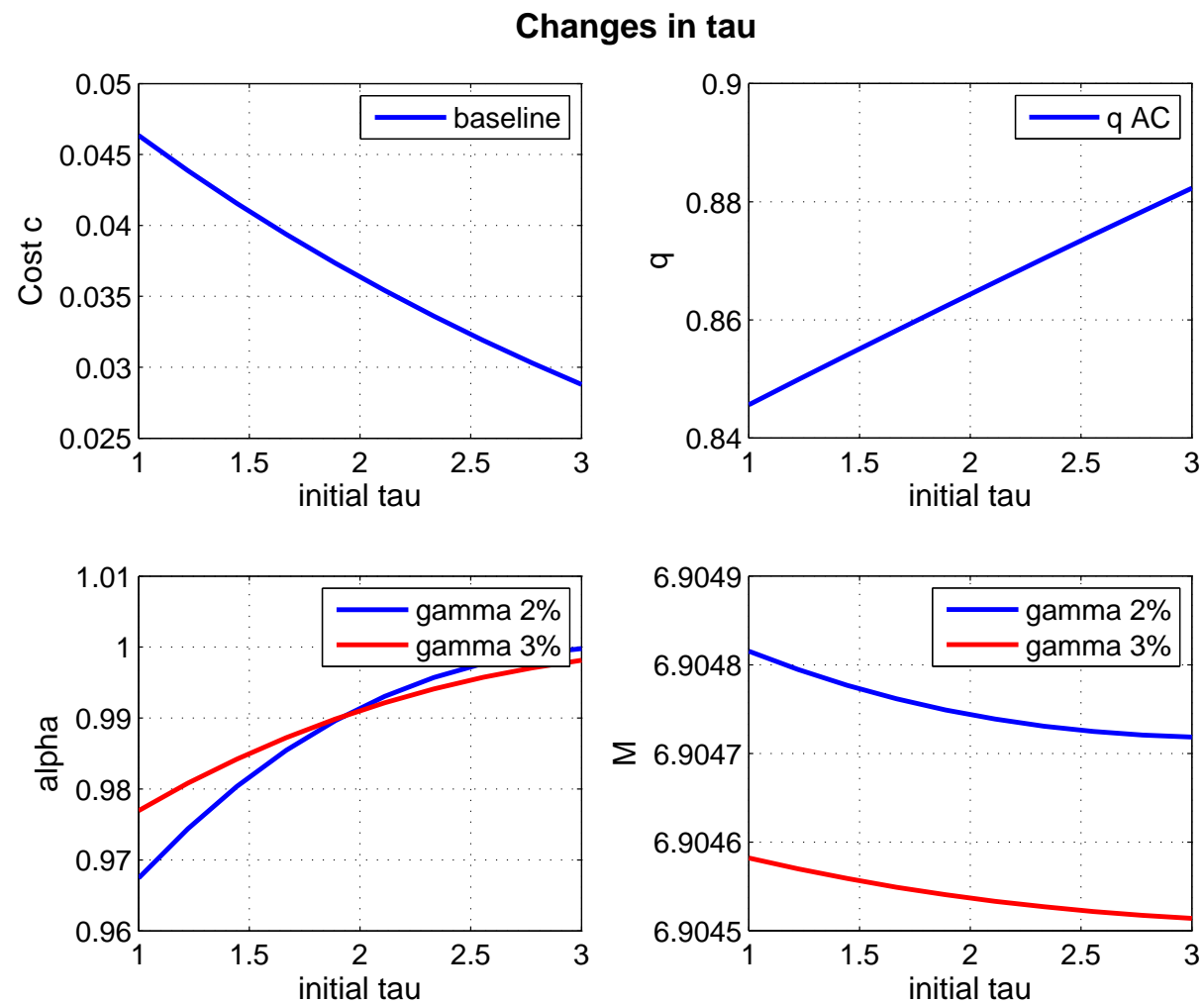

values of the non-linear term in the cost function. However, for high values of $\tau$ the share of innovative firms in the market is higher in the slow growing economy than in the benchmark economy.

Figure 11 shows the response of the cost parameter to changes in the curvature of the profit function. The cost parameter decreases in the span of control (higher $\theta$ ). The difference in the share of innovative firms in the market between the benchmark and the slow growth economy displays a similar dynamic as the one depicted for changes in the probability of success. When profits are large (low $\theta$ ) the share of innovative firms in the slow growing economy is smaller than in the high growth economy. The same is true for relatively high values of $\theta$, as it is the case in our benchmark calibration. For 
Figure 11: Comparative statics, changes in the curvature of the profit function.
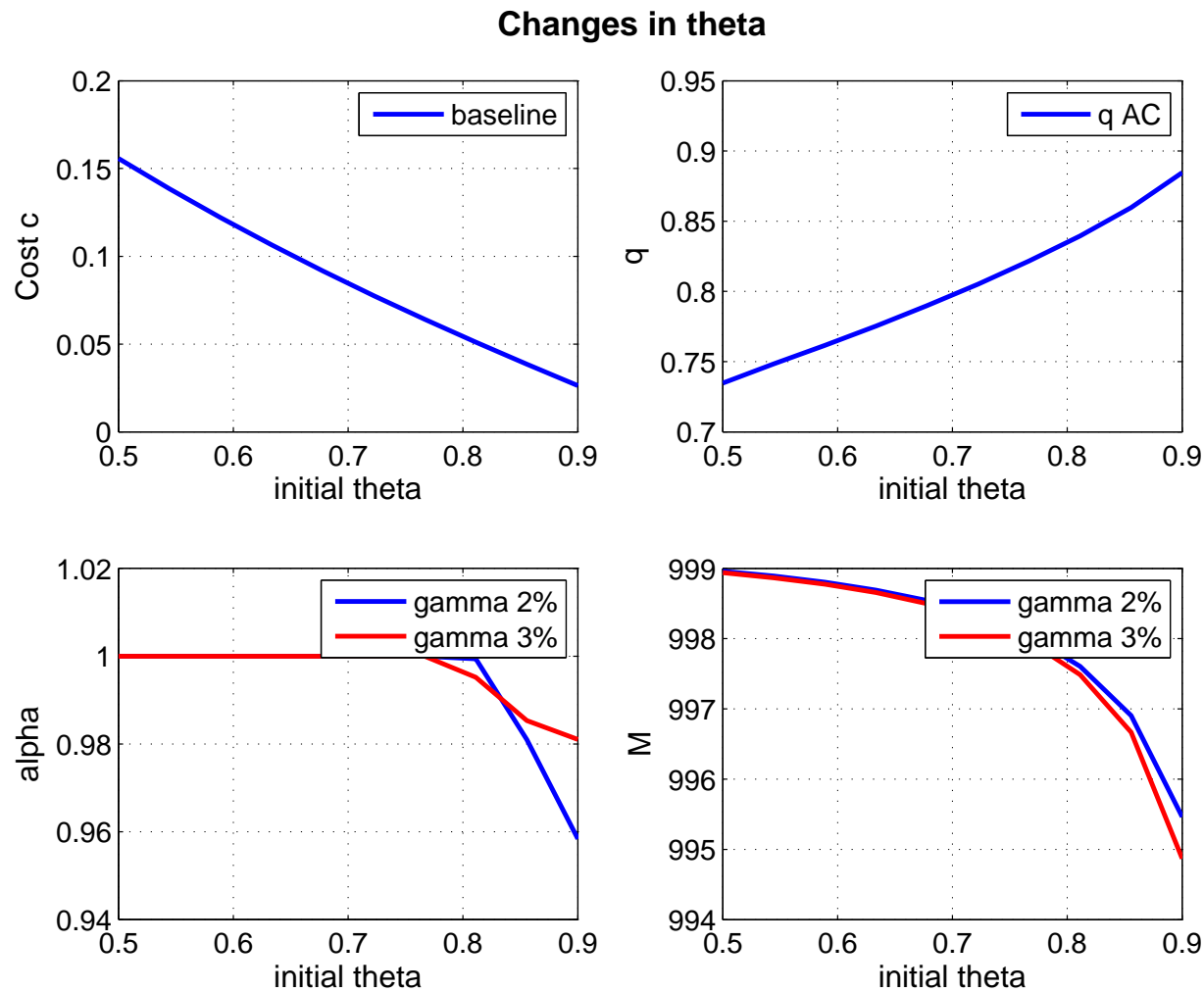

intermediate levels of the span of control the share of innovative firms in the market can be higher in the low growth economy. A similar dynamic is observed for the measure of firms operating in the market.

It is important to highlight that the disparate impact on the measure of firms observed when shifting $\theta$ and $q$ is important for the model predictions in terms of total factor productivity. When the measure of firms increases in the slow growth economy, and the share of innovative firms drops, then TFP is slower than in the benchmark economy. However, when the measure of firms operating in the slow growth economy is lower than in the benchmark economy, it is possible for TFP to be higher in the slow growth economy. A lower measure of operating firms induces savings in overhead costs 
that might compensate for the lower measure of innovative firms in the market.

\section{Extension: Idiosyncratic investment returns}

In our benchmark economy, firms' productivity and sales can grow in time. However, their employment level does not grow. How is this possible? Along the balanced growth path, innovative firms grow at a rate $\phi$, which is the rate of growth of average productivity in the market. Hence, if a firm is successful in innovating, it is able to sustain a given level of employment. If it does not, its employment level shrinks. In other words, if a firm is born with a position in the distribution of productivities relative to the mean (which determines their employment), it can never step ahead in the ordering. This is an undesirable feature, as productivity growth and employment growth correlate positively in the data.

In this section, we augment the benchmark economy to allow for i.i.d. shocks on the returns to firm's investment, whenever the firm is successful. Intuitively, a firm invests in $\mathrm{R} \& \mathrm{D}$ expecting on average a growth rate of $\exp (\phi d t)$, but when the project is realized, the actual growth can be below or above average.

Because the shocks are assumed i.i.d., the value functions for innovative and noninnovative firms are linear in $z^{\eta}$. However, optimal investment in growth is now a function of the idiosyncratic shocks. Computing the optimality conditions of the household, we obtain

$$
z^{\prime}=\exp \left(\phi^{\varepsilon} d t\right) z=\left(\exp (\widehat{\beta} d t) \frac{1}{c \tau} q B_{I} \frac{\eta}{\eta+1} \frac{\bar{\varepsilon}^{\eta+1}-\underline{\varepsilon}^{\eta+1}}{\bar{\varepsilon}-\underline{\varepsilon}}\right)^{\frac{1}{\tau-\eta}} .
$$

Given the investment strategy of the firms, BGP is preserved whenever there is an invariant distribution of firms in the innovative sector. In this model, it is a Pareto distribution. The existence of an invariant measure allows us to show that the measures of firms in each sector are non-degenerate and constant in time. 
Proposition 5 Suppose that, conditional on innovating, firms receive an i.i.d. shock, $\varepsilon$, that is uniformly distributed over $(\underline{\varepsilon}, \bar{\varepsilon})$. Suppose also that the shocks satisfy $\bar{\varepsilon}^{\lambda+1}>$ $\bar{\varepsilon}(\lambda+1)-\lambda$. If the initial distribution of productivity is Pareto with parameter $\lambda$, the invariant distribution of innovative firms in the market is approximately Pareto with parameter $\lambda$. Furthermore, the endogenous growth rate of the threshold for switching to the non-innovative sector grows at rate $\mu^{*}>\phi$ that solves

$$
\frac{\left(\bar{\varepsilon}^{\lambda+1}\right)}{e^{\left(\mu^{*}-\phi\right) d t}}=\bar{\varepsilon}(\lambda+1)-\lambda e^{\left(\mu^{*}-\phi\right) d t}
$$

Hence, the BGP is preserved. Differently from before, the rate of selection in the market, measured by the growth rate of the switching threshold towards the noninnovative sector, depends on the behavior of the best firms in the market, through the upper threshold $\bar{\varepsilon}$.

Next, we show average employment-age profiles, and a histogram with the age distribution for our simulated economy. We parameterize our economy as in the baseline calibration presented in the previous section. We set the support for idiosyncratic shocks to $(\underline{\varepsilon}, \bar{\varepsilon})=(0.75,1.25)$. We simulate paths for 1000 firms over 100 periods. We solve for the thresholds of productivity in the first period by normalizing the first wage to unity, and solve for the path of wages and thresholds across 100 periods. Consistently with our model, we replace an exiting firm with a new one with productivity drawn from the distribution of incumbents in that particular period.

The left panel of Figure 12 presents a quadratic fit to average employment per age as predicted by the model. Younger firms are on average smaller than older firms. The quadratic fit of the employment-age profiles is more hump-shaped than the profiles observed in the data for high-income economies. However, the magnitude of the relative sizes of firms is very close to the one in the data. The predicted age distribution has very few small firms relative to the data (see the right panel of Figure 12). In other words, for the baseline parametrization, firms either (i) enter the market relatively large or (ii) the success rate is relatively high, which would explain high survival. We explore 
these possibilities by looking at comparative statics across various specifications.

Figure 12: Employment age profile (left) and age distribution, benchmark
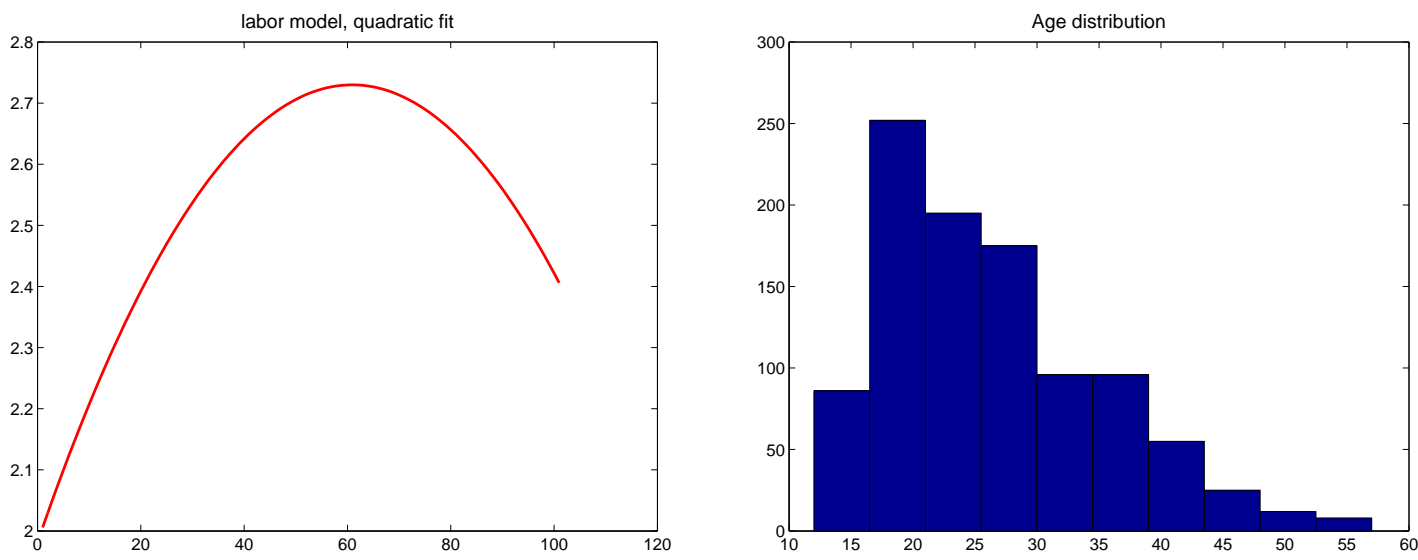

When we decrease the probability of success to $q=0.3$, the average age of firms in the market decreases. In this case, the oldest firms in the market are about 35 years old, and the age distribution loses its exponential shape. Both the left and right tails become thinner (see the right panel of Figure 13). More importantly, the left panel of Figure 13 shows that the employment-age profile flips.

Next, we increase the support of the shocks to $(\underline{\varepsilon}, \bar{\varepsilon})=(0.5,1.5)$. The main difference with the benchmark economy is the predicted age distribution, that shifts toward younger firms (right panel, Figure 14). If the change in the support of shocks is asymmetric, i.e. $(\underline{\varepsilon}, \bar{\varepsilon})=(0.5,1.25)$, the employment-age profiles get flatter (left panel, Figure 15) and the size distribution shifts toward younger firms (right panel, Figure $15)$. 
Figure 13: Employment age profile (left) and age distribution, low probability of success $(q=0.3)$
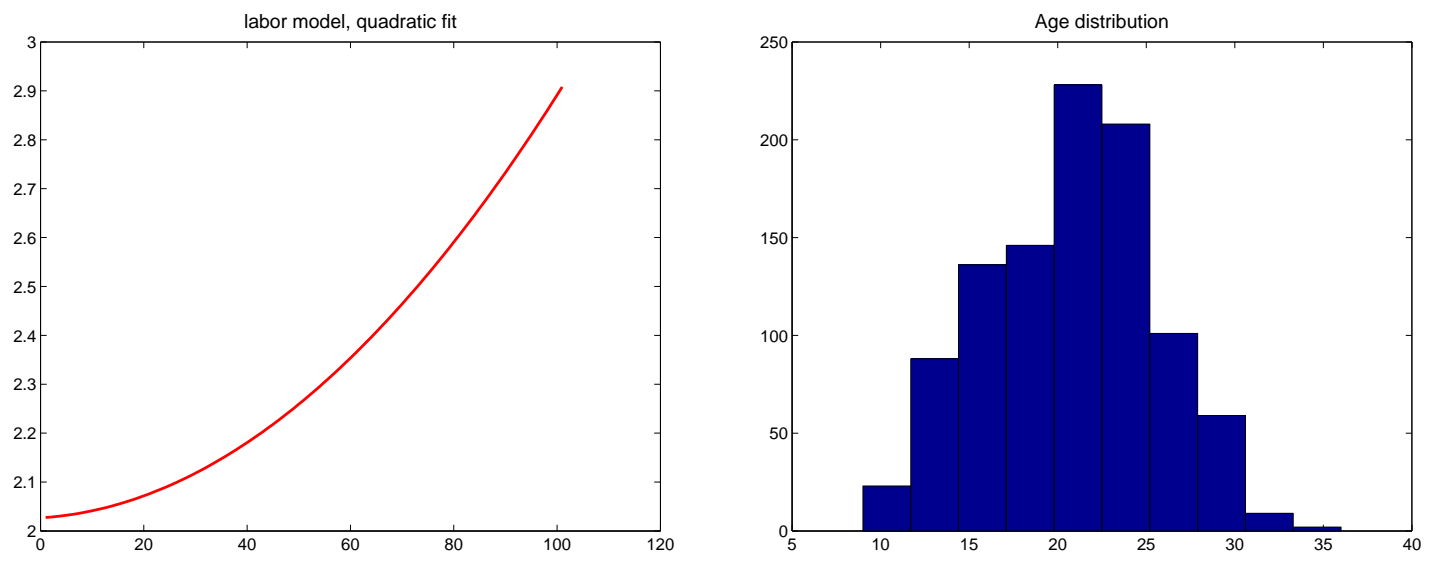

Figure 14: Employment age profile (left) and age distribution, alternative shocks
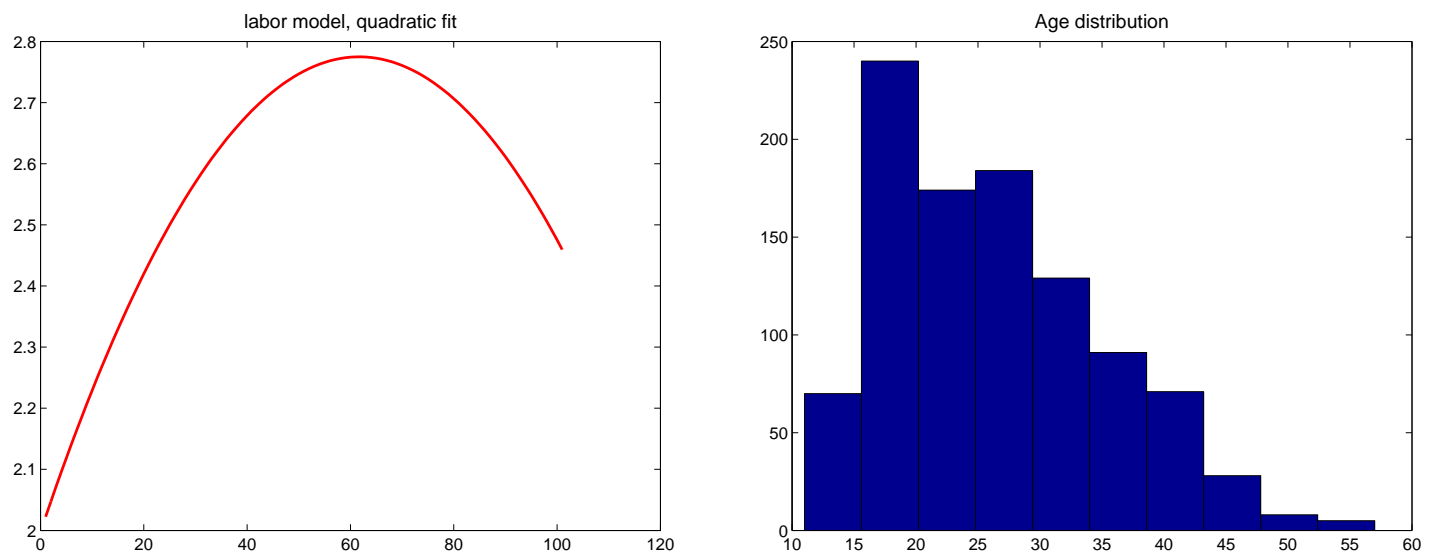
Figure 15: Employment age profile (left) and age distribution, asymmetric shocks
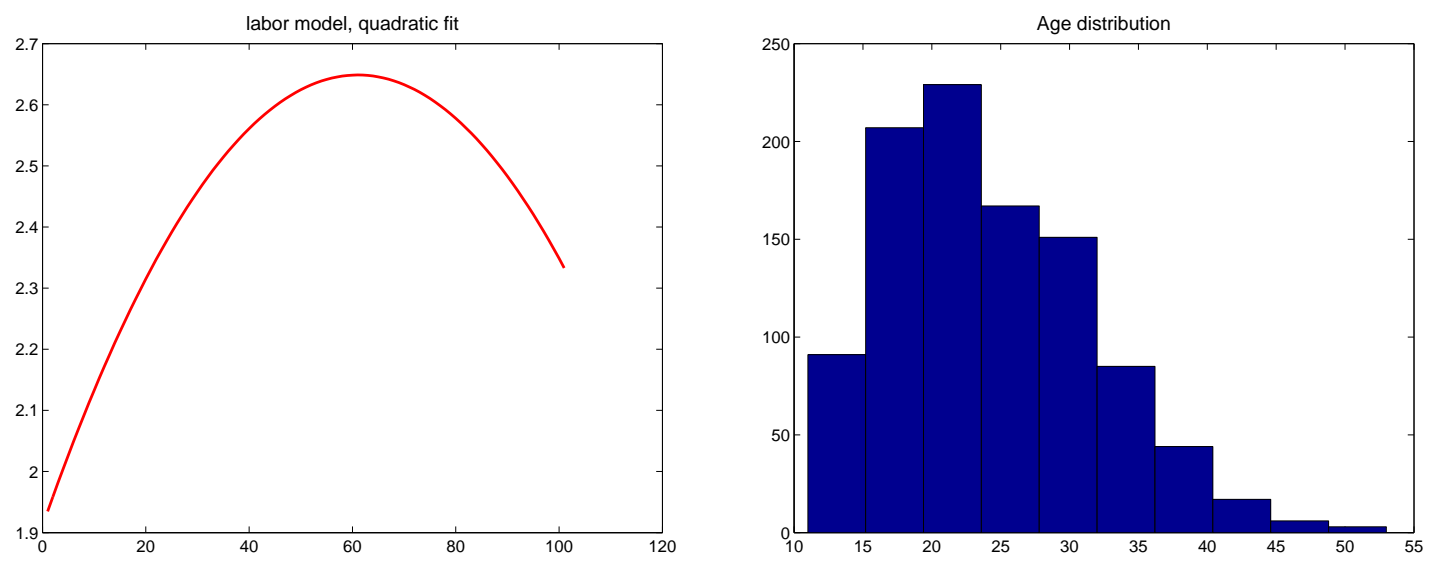


\section{Final remarks}

We build a stylized model of firm dynamics with growth where the aggregate growth of the economy is linked to the probability of success that firms face in their returns to investment in firm growth.

In particular, our model highlights two channels through which such probability affects firm-level as well as aggregate growth. First, lower probability of success has an impact on firm growth directly by decreasing the frequency of successful growth episodes in a firm's life cycle, and indirectly by lowering incentives to invest in growth. This partial equilibrium mechanism, increases the firm-level and aggregate growth in economies with higher probability of success.

What is also relevant, and helpful to fit important features in the data, is a general equilibrium channel. Economies with higher probability of success in turning investments into actual growth will have higher growth, making survival harder. Average productivity will grow faster, and the distribution of productivity will shift upwards, possibly preserving its shape. This feature enables the economy we study in this paper to replicate the lack of systematic relationship between the tail of the size distribution and growth.

Firm level uncertainty is built into our benchmark model only through the probability of realizing positive returns to investment. In order to see the link between such probability of success and the firm level patterns of employment growth, we extend our benchmark economy allowing for return uncertainty. We are able to generate employment age patterns comparable to those in the data. Our results suggest that high

probability of success increases the average age of firms in the economy and makes the right tail of the employment distribution thicker. 


\section{References}

Akcigit, U., H. Alp, And M. Peters (2014): "Lack of Selection and Imperfect Managerial Contracts: Firm Dynamics in Developing Countries," Manuscript.

Atkeson, A., and A. T. Burstein (2010): "Innovation, Firm Dynamics, and International Tradelll," Journal of Political Economy, 118(3), 433-484.

Atkeson, A., And P. J. Kehoe (2005): "Modeling and Measuring Organization Capital," Journal of Political Economy, University of Chicago Press, 113(5), 10261053.

Axtell, R. (2001): "Zipf Distribution of U.S. Firm Sizes," Science.

Bartelsman, E., J. Haltiwanger, and S. Scarpetta (2009): "Measuring and Analyzing Cross-country Differences in Firm Dynamics," in Producer Dynamics: New Evidence from Micro Data, NBER Chapters, pp. 15-76. National Bureau of Economic Research, Inc.

Bloom, N., And R. SAdun (2012): "The Organization of Firms Across Countries," The Quarterly Journal of Economics, 127(4), 1663-1705.

Carvalho, V., And X. Gabaix (2013): "The Great Diversification and Its Undoing," American Economic Review, 103(5), 1697-1727.

Christensen, C. (ed.) (1997): The Innovators Dilemma. Harvard Business Review Press.

Cole, H. L., J. Greenwood, and J. M. Sanchez (2012): "Why Doesn't Technology Flow from Rich to Poor Countries?," Working Papers, Federal Reserve Bank of St. Louis 2012-040, Federal Reserve Bank of St. Louis. 
Da-Rocha, J. M., M. M. Tavares, and D. Restuccia (2014): "Policy Distortions and Aggregate Productivity with Endogenous Establishment-Level Productivity," Working Papers tecipa-523, University of Toronto, Department of Economics.

Eslava, M., J. Haltiwanger, A. Kugler, and M. Kugler (2004): "The effects of structural reforms on productivity and profitability enhancing reallocation: evidence from Colombia," Journal of Development Economics, 75(2), 333-371.

Hsien, C.-T., And P. J. KLenow (2009): "Misallocation and Manufacturing TFP in China and India," The Quarterly Journal of Economics, 124(4), 1403-1448.

(2012): "The Life Cycle of Plants in India and Mexico," NBER Working Papers 18133, National Bureau of Economic Research, Inc.

Klette, T. J., and S. Kortum (2004): "Innovating Firms and Aggregate Innovation," Journal of Political Economy, 112(5), 986-1018.

Knack, S., And P. Keefer (1997): "Does Social Capital Have an Economic Payoff? A Cross-Country Investigation," The Quarterly Journal of Economics, 112(4), 125188.

Luttmer, E. G. (2010): "On the mechanics of firm growth," Discussion paper.

Perla, J., and C. Tonetti (2014): "Equilibrium Imitation and Growth," Journal of Political Economy, 122(1), $52-76$.

Peters, M. (2011): "Heterogeneous Mark-Ups and Endogenous Misallocation," Discussion paper.

Restuccia, D., and R. Rogerson (2008): "Policy Distortions and Aggregate Productivity with Heterogeneous Plants," Review of Economic Dynamics, 11(4), 707720. 
Roberts, M. J., and J. R. Tybout (1997): "Producer Turnover and Productivity Growth in Developing Countries," World Bank Research Observer, 12(1), 1-18.

Thomke, S. (ed.) (2003): Experimentation Matters: Unlocking the Potential of New Technologies for Innovation. Harvard Business School Press. 


\section{Appendix A}

Proposition 1 (Proof). We first argue that $B_{I}>B_{N \hat{t}}$.

$$
\begin{gathered}
B_{I}=\max _{\phi \geq 1}(1-\theta) \theta^{\frac{\theta}{1-\theta}}-c e^{\phi \tau d t} \\
+e^{\widehat{\beta} d t}\left[q B_{I} e^{\phi \eta d t}+(1-q) B_{I}\right] \\
\geq(1-\theta) \theta^{\frac{\theta}{1-\theta}}-c+e^{\widehat{\beta} d t} B_{I}
\end{gathered}
$$

Meanwhile,

$$
B_{N \hat{t}}<(1-\theta) \theta^{\frac{\theta}{1-\theta}}+e^{\widehat{\beta} d t} B_{N \hat{t}}
$$

Hence for $c$ small enough,

$$
B_{I} \geq \frac{(1-\theta) \theta^{\frac{\theta}{1-\theta}}-c}{1-e^{\widehat{\beta} d t}}>B_{N \hat{t}}
$$

We also know that $D_{I}>D_{N T}$ since

$$
D_{I}-D_{N T}=f\left(1-e^{\widehat{\widehat{\beta}}(T-1)}\right)
$$

$\widehat{\widehat{\beta}}=-(\delta+r+\gamma)$

Now we use the last findings to show that the surplus is increasing in unsuccessful innovations, in other words it decreases with $T(z, w)$. Define the surplus function:

$$
s(z, w) \equiv-V_{I}(z, w)+W_{N T}(z, w)=-\left(B_{I}-B_{N T}\right) \frac{z^{\eta}}{w^{\frac{\theta}{1-\theta}}}+\left(D_{I}-D_{N T}\right) w
$$


Then in one unsuccessful innovation the surplus becomes:

$$
\begin{gathered}
s\left(z, w e^{\gamma d t}\right)=-\left(B_{I}-B_{N T-1}\right) \frac{z^{\eta}}{\left(w e^{\gamma d t}\right)^{\frac{\theta}{1-\theta}}}+\left(D_{I}-D_{N T-1}\right) w e^{\gamma d t} \\
=-\left(B_{I}-\left(B_{N T}-(1-\theta) \theta^{\frac{\theta}{1-\theta}} e^{\widehat{\beta} T}\right)\right) \frac{z^{\eta}}{\left(w e^{\gamma d t}\right)^{\frac{\theta}{1-\theta}}}+\left(D_{I}-\left(D_{N T}-f e^{\widehat{\widehat{\beta}} T}\right)\right) w e^{\gamma t} \\
s(z, w \gamma)-s(z, w)=-\left(B_{I}-B_{N T}\right) \frac{z^{\eta}}{w^{\frac{\theta}{1-\theta}}}\left(e^{-\frac{\theta}{1-\theta} \gamma d t}-1\right)+\left(D_{I}-D_{N T}\right) w\left(e^{\gamma d t}-1\right) \\
-\frac{z^{\eta}}{\left(w e^{\gamma d t}\right)^{\frac{\theta}{1-\theta}}}(1-\theta) \theta^{\frac{\theta}{1-\theta}} e^{\widehat{\beta} T}+f e^{\widehat{\widehat{\beta}} T} w e^{\gamma d t} \\
s(z, w \gamma)-s(z, w)=-\left(B_{I}-B_{N T}\right) \frac{z^{\eta}}{w^{\frac{\theta}{1-\theta}}}\left(e^{-\frac{\theta}{1-\theta} \gamma d t}-1\right)+\left(D_{I}-D_{N T}\right) w\left(e^{\gamma d t}-1\right) \\
-e^{-(\delta+r) T}\left[(1-\theta) \theta^{\frac{\theta}{1-\theta}} \frac{z^{\eta}}{(w)^{\frac{\theta}{1-\theta}}}-f w\right]
\end{gathered}
$$

We know $\gamma>0, B_{I}>B_{N T}$ and $D_{I}>D_{N T}$. Hence the first line in the equation below is positive. Moreover, by definition of $T$,

We know that for $z$ and $w$ such that $T(z, w)=0$, we have

$$
\begin{gathered}
(1-\theta) \theta^{\frac{\theta}{1-\theta}} \frac{z^{\eta}}{w^{\frac{\theta}{1-\theta}}}-f w \leq 0 \\
W_{N 0}=0
\end{gathered}
$$

Hence $s(z, w \gamma)-s(z, w)$ is positive. This shows that the surplus increases in every unsuccessful innovation. Also,

$$
V_{I}(z, w)=(1-\theta) \theta^{\frac{\theta}{1-\theta}} \frac{z^{\eta}}{w^{\frac{\theta}{1-\theta}}}-c \frac{e^{\phi \tau d t} z^{\eta}}{w^{\frac{\theta}{1-\theta}}}-f w<0 .
$$

So for $T=0$ the surplus is positive. Moreover,

$$
\lim _{T \rightarrow \infty} D_{N T}=D_{I}
$$


and

$$
B_{N T}<B_{I}
$$

so that there is large enough $T$ such that the surplus is negative. This shows that there exists $\hat{t}<\infty$ such that an $I$-firm sells the technology to an entrant $N$ firm.

Proposition 1 (Proof). We first argue that $B_{I}>B_{N \hat{t}}$.

$$
\begin{gathered}
B_{I}=\max _{\phi \geq 1}(1-\theta) \theta^{\frac{\theta}{1-\theta}}-c e^{\phi \tau d t} \\
+e^{\widehat{\beta} d t}\left[q B_{I} e^{\phi \eta d t}+(1-q) B_{I}\right] \\
\geq(1-\theta) \theta^{\frac{\theta}{1-\theta}}-c+e^{\widehat{\beta} d t} B_{I}
\end{gathered}
$$

Meanwhile,

$$
B_{N \hat{t}}<(1-\theta) \theta^{\frac{\theta}{1-\theta}}+e^{\widehat{\beta} d t} B_{N \hat{t}}
$$

Hence for $c$ small enough,

$$
B_{I} \geq \frac{(1-\theta) \theta^{\frac{\theta}{1-\theta}}-c}{1-e^{\widehat{\beta} d t}}>B_{N \hat{t}} .
$$

We also know that $D_{I}>D_{N T}$ since

$$
D_{I}-D_{N T}=f\left(1-e^{\widehat{\widehat{\beta}}(T-1)}\right)
$$

$\widehat{\widehat{\beta}}=-(\delta+r+\gamma)$

Now we use the last findings to show that the surplus is increasing in unsuccessful innovations, in other words it decreases with $T(z, w)$. Define the surplus function:

$$
s(z, w) \equiv-V_{I}(z, w)+W_{N T}(z, w)=-\left(B_{I}-B_{N T}\right) \frac{z^{\eta}}{w^{\frac{\theta}{1-\theta}}}+\left(D_{I}-D_{N T}\right) w
$$


Then in one unsuccessful innovation the surplus becomes:

$$
\begin{gathered}
s\left(z, w e^{\gamma d t}\right)=-\left(B_{I}-B_{N T-1}\right) \frac{z^{\eta}}{\left(w e^{\gamma d t}\right)^{\frac{\theta}{1-\theta}}}+\left(D_{I}-D_{N T-1}\right) w e^{\gamma d t} \\
=-\left(B_{I}-\left(B_{N T}-(1-\theta) \theta^{\frac{\theta}{1-\theta}} e^{\widehat{\beta} T}\right)\right) \frac{z^{\eta}}{\left(w e^{\gamma d t}\right)^{\frac{\theta}{1-\theta}}}+\left(D_{I}-\left(D_{N T}-f e^{\widehat{\widehat{\beta}} T}\right)\right) w e^{\gamma t} \\
s(z, w \gamma)-s(z, w)=-\left(B_{I}-B_{N T}\right) \frac{z^{\eta}}{w^{\frac{\theta}{1-\theta}}}\left(e^{-\frac{\theta}{1-\theta} \gamma d t}-1\right)+\left(D_{I}-D_{N T}\right) w\left(e^{\gamma d t}-1\right) \\
-\frac{z^{\eta}}{\left(w e^{\gamma d t}\right)^{\frac{\theta}{1-\theta}}}(1-\theta) \theta^{\frac{\theta}{1-\theta}} e^{\widehat{\beta} T}+f e^{\widehat{\widehat{\beta}} T} w e^{\gamma d t} \\
s(z, w \gamma)-s(z, w)=-\left(B_{I}-B_{N T}\right) \frac{z^{\eta}}{w^{\frac{\theta}{1-\theta}}}\left(e^{-\frac{\theta}{1-\theta} \gamma d t}-1\right)+\left(D_{I}-D_{N T}\right) w\left(e^{\gamma d t}-1\right) \\
-e^{-(\delta+r) T}\left[(1-\theta) \theta^{\frac{\theta}{1-\theta}} \frac{z^{\eta}}{(w)^{\frac{\theta}{1-\theta}}}-f w\right]
\end{gathered}
$$

We know $\gamma>0, B_{I}>B_{N T}$ and $D_{I}>D_{N T}$. Hence the first line in the equation below is positive. Moreover, by definition of $T$,

We know that for $z$ and $w$ such that $T(z, w)=0$, we have

$$
\begin{gathered}
(1-\theta) \theta^{\frac{\theta}{1-\theta}} \frac{z^{\eta}}{w^{\frac{\theta}{1-\theta}}}-f w \leq 0 \\
W_{N 0}=0
\end{gathered}
$$

Hence $s(z, w \gamma)-s(z, w)$ is positive. This shows that the surplus increases in every unsuccessful innovation. Also,

$$
V_{I}(z, w)=(1-\theta) \theta^{\frac{\theta}{1-\theta}} \frac{z^{\eta}}{w^{\frac{\theta}{1-\theta}}}-c \frac{e^{\phi \tau d t} z^{\eta}}{w^{\frac{\theta}{1-\theta}}}-f w<0 .
$$

So for $T=0$ the surplus is positive. Moreover,

$$
\lim _{T \rightarrow \infty} D_{N T}=D_{I}
$$


and

$$
B_{N T}<B_{I}
$$

so that there is large enough $T$ such that the surplus is negative. This shows that there exists $\hat{t}<\infty$ such that an $I$-firm sells the technology to an entrant $N$ firm.

Proposition 1 (Proof). We first argue that $B_{I}>B_{N \hat{t}}$.

$$
\begin{gathered}
B_{I}=\max _{\phi \geq 1}(1-\theta) \theta^{\frac{\theta}{1-\theta}}-c e^{\phi \tau d t} \\
+e^{\widehat{\beta} d t}\left[q B_{I} e^{\phi \eta d t}+(1-q) B_{I}\right] \\
\geq(1-\theta) \theta^{\frac{\theta}{1-\theta}}-c+e^{\widehat{\beta} d t} B_{I}
\end{gathered}
$$

Meanwhile,

$$
B_{N \hat{t}}<(1-\theta) \theta^{\frac{\theta}{1-\theta}}+e^{\widehat{\beta} d t} B_{N \hat{t}}
$$

Hence for $c$ small enough,

$$
B_{I} \geq \frac{(1-\theta) \theta^{\frac{\theta}{1-\theta}}-c}{1-e^{\widehat{\beta} d t}}>B_{N \hat{t}} .
$$

We also know that $D_{I}>D_{N T}$ since

$$
D_{I}-D_{N T}=f\left(1-e^{\widehat{\widehat{\beta}}(T-1)}\right)
$$

$\widehat{\widehat{\beta}}=-(\delta+r+\gamma)$

Now we use the last findings to show that the surplus is increasing in unsuccessful innovations, in other words it decreases with $T(z, w)$. Define the surplus function:

$$
s(z, w) \equiv-V_{I}(z, w)+W_{N T}(z, w)=-\left(B_{I}-B_{N T}\right) \frac{z^{\eta}}{w^{\frac{\theta}{1-\theta}}}+\left(D_{I}-D_{N T}\right) w
$$


Then in one unsuccessful innovation the surplus becomes:

$$
\begin{gathered}
s\left(z, w e^{\gamma d t}\right)=-\left(B_{I}-B_{N T-1}\right) \frac{z^{\eta}}{\left(w e^{\gamma d t}\right)^{\frac{\theta}{1-\theta}}}+\left(D_{I}-D_{N T-1}\right) w e^{\gamma d t} \\
=-\left(B_{I}-\left(B_{N T}-(1-\theta) \theta^{\frac{\theta}{1-\theta}} e^{\widehat{\beta} T}\right)\right) \frac{z^{\eta}}{\left(w e^{\gamma d t}\right)^{\frac{\theta}{1-\theta}}}+\left(D_{I}-\left(D_{N T}-f e^{\widehat{\widehat{\beta}} T}\right)\right) w e^{\gamma t} \\
s(z, w \gamma)-s(z, w)=-\left(B_{I}-B_{N T}\right) \frac{z^{\eta}}{w^{\frac{\theta}{1-\theta}}}\left(e^{-\frac{\theta}{1-\theta} \gamma d t}-1\right)+\left(D_{I}-D_{N T}\right) w\left(e^{\gamma d t}-1\right) \\
-\frac{z^{\eta}}{\left(w e^{\gamma d t}\right)^{\frac{\theta}{1-\theta}}}(1-\theta) \theta^{\frac{\theta}{1-\theta}} e^{\widehat{\beta} T}+f e^{\widehat{\widehat{\beta}} T} w e^{\gamma d t} \\
s(z, w \gamma)-s(z, w)=-\left(B_{I}-B_{N T}\right) \frac{z^{\eta}}{w^{\frac{\theta}{1-\theta}}}\left(e^{-\frac{\theta}{1-\theta} \gamma d t}-1\right)+\left(D_{I}-D_{N T}\right) w\left(e^{\gamma d t}-1\right) \\
-e^{-(\delta+r) T}\left[(1-\theta) \theta^{\frac{\theta}{1-\theta}} \frac{z^{\eta}}{(w)^{\frac{\theta}{1-\theta}}}-f w\right]
\end{gathered}
$$

We know $\gamma>0, B_{I}>B_{N T}$ and $D_{I}>D_{N T}$. Hence the first line in the equation below is positive. Moreover, by definition of $T$,

We know that for $z$ and $w$ such that $T(z, w)=0$, we have

$$
\begin{gathered}
(1-\theta) \theta^{\frac{\theta}{1-\theta}} \frac{z^{\eta}}{w^{\frac{\theta}{1-\theta}}}-f w \leq 0 \\
W_{N 0}=0
\end{gathered}
$$

Hence $s(z, w \gamma)-s(z, w)$ is positive. This shows that the surplus increases in every unsuccessful innovation. Also,

$$
V_{I}(z, w)=(1-\theta) \theta^{\frac{\theta}{1-\theta}} \frac{z^{\eta}}{w^{\frac{\theta}{1-\theta}}}-c \frac{e^{\phi \tau d t} z^{\eta}}{w^{\frac{\theta}{1-\theta}}}-f w<0 .
$$

So for $T=0$ the surplus is positive. Moreover,

$$
\lim _{T \rightarrow \infty} D_{N T}=D_{I}
$$


and

$$
B_{N T}<B_{I}
$$

so that there is large enough $T$ such that the surplus is negative. This shows that there exists $\hat{t}<\infty$ such that an $I$-firm sells the technology to an entrant $N$ firm.

Proposition 1 (Proof). We first argue that $B_{I}>B_{N \hat{t}}$.

$$
\begin{gathered}
B_{I}=\max _{\phi \geq 1}(1-\theta) \theta^{\frac{\theta}{1-\theta}}-c e^{\phi \tau d t} \\
+e^{\widehat{\beta} d t}\left[q B_{I} e^{\phi \eta d t}+(1-q) B_{I}\right] \\
\geq(1-\theta) \theta^{\frac{\theta}{1-\theta}}-c+e^{\widehat{\beta} d t} B_{I}
\end{gathered}
$$

Meanwhile,

$$
B_{N \hat{t}}<(1-\theta) \theta^{\frac{\theta}{1-\theta}}+e^{\widehat{\beta} d t} B_{N \hat{t}}
$$

Hence for $c$ small enough,

$$
B_{I} \geq \frac{(1-\theta) \theta^{\frac{\theta}{1-\theta}}-c}{1-e^{\widehat{\beta} d t}}>B_{N \hat{t}} .
$$

We also know that $D_{I}>D_{N T}$ since

$$
D_{I}-D_{N T}=f\left(1-e^{\widehat{\widehat{\beta}}(T-1)}\right)
$$

$\widehat{\widehat{\beta}}=-(\delta+r+\gamma)$

Now we use the last findings to show that the surplus is increasing in unsuccessful innovations, in other words it decreases with $T(z, w)$. Define the surplus function:

$$
s(z, w) \equiv-V_{I}(z, w)+W_{N T}(z, w)=-\left(B_{I}-B_{N T}\right) \frac{z^{\eta}}{w^{\frac{\theta}{1-\theta}}}+\left(D_{I}-D_{N T}\right) w
$$


Then in one unsuccessful innovation the surplus becomes:

$$
\begin{gathered}
s\left(z, w e^{\gamma d t}\right)=-\left(B_{I}-B_{N T-1}\right) \frac{z^{\eta}}{\left(w e^{\gamma d t}\right)^{\frac{\theta}{1-\theta}}}+\left(D_{I}-D_{N T-1}\right) w e^{\gamma d t} \\
=-\left(B_{I}-\left(B_{N T}-(1-\theta) \theta^{\frac{\theta}{1-\theta}} e^{\widehat{\beta} T}\right)\right) \frac{z^{\eta}}{\left(w e^{\gamma d t}\right)^{\frac{\theta}{1-\theta}}}+\left(D_{I}-\left(D_{N T}-f e^{\widehat{\widehat{\beta}} T}\right)\right) w e^{\gamma t} \\
s(z, w \gamma)-s(z, w)=-\left(B_{I}-B_{N T}\right) \frac{z^{\eta}}{w^{\frac{\theta}{1-\theta}}}\left(e^{-\frac{\theta}{1-\theta} \gamma d t}-1\right)+\left(D_{I}-D_{N T}\right) w\left(e^{\gamma d t}-1\right) \\
-\frac{z^{\eta}}{\left(w e^{\gamma d t}\right)^{\frac{\theta}{1-\theta}}}(1-\theta) \theta^{\frac{\theta}{1-\theta}} e^{\widehat{\beta} T}+f e^{\widehat{\widehat{\beta}} T} w e^{\gamma d t} \\
s(z, w \gamma)-s(z, w)=-\left(B_{I}-B_{N T}\right) \frac{z^{\eta}}{w^{\frac{\theta}{1-\theta}}}\left(e^{-\frac{\theta}{1-\theta} \gamma d t}-1\right)+\left(D_{I}-D_{N T}\right) w\left(e^{\gamma d t}-1\right) \\
-e^{-(\delta+r) T}\left[(1-\theta) \theta^{\frac{\theta}{1-\theta}} \frac{z^{\eta}}{(w)^{\frac{\theta}{1-\theta}}}-f w\right]
\end{gathered}
$$

We know $\gamma>0, B_{I}>B_{N T}$ and $D_{I}>D_{N T}$. Hence the first line in the equation below is positive. Moreover, by definition of $T$,

We know that for $z$ and $w$ such that $T(z, w)=0$, we have

$$
\begin{gathered}
(1-\theta) \theta^{\frac{\theta}{1-\theta}} \frac{z^{\eta}}{w^{\frac{\theta}{1-\theta}}}-f w \leq 0 \\
W_{N 0}=0
\end{gathered}
$$

Hence $s(z, w \gamma)-s(z, w)$ is positive. This shows that the surplus increases in every unsuccessful innovation. Also,

$$
V_{I}(z, w)=(1-\theta) \theta^{\frac{\theta}{1-\theta}} \frac{z^{\eta}}{w^{\frac{\theta}{1-\theta}}}-c \frac{e^{\phi \tau d t} z^{\eta}}{w^{\frac{\theta}{1-\theta}}}-f w<0 .
$$

So for $T=0$ the surplus is positive. Moreover,

$$
\lim _{T \rightarrow \infty} D_{N T}=D_{I}
$$


and

$$
B_{N T}<B_{I}
$$

so that there is large enough $T$ such that the surplus is negative. This shows that there exists $\hat{t}<\infty$ such that an $I$-firm sells the technology to an entrant $N$ firm.

\section{Proposition 3 (Proof).}

Define:

$$
\begin{gathered}
X_{I} \equiv E_{I}\left(z^{\eta}\right) \\
X_{N} \equiv E_{N}\left(z^{\eta}\right)
\end{gathered}
$$

Also define the cumulative distribution function for the group $I$ as:

$$
F_{I}(z)= \begin{cases}1-\left(\frac{\hat{z}}{z}\right)^{\lambda}, & \text { if } z \geq \hat{z} \\ 0, & \mathrm{o} / \mathrm{w}\end{cases}
$$

Moreover, let $M_{I}$ and $M_{N}$ be the number of firms in each group, which will be constant in the BGP, let $\alpha \equiv \frac{M_{I}}{M_{I}+M_{N}}$. To show that $\phi=\mu$ we first show that $\mu \leq \phi$ along the BGP. Define $\bar{z} \equiv \max \left\{\hat{z}, e^{\mu-\phi} \hat{z}\right\}$, and $a \equiv \frac{\hat{z}}{\bar{z}}$. Then

$$
\begin{aligned}
X_{I}^{\prime}=(1- & \delta) q \int_{\bar{z}}^{\infty}\left(e^{\phi d t} z\right)^{\eta} \frac{\lambda \tilde{z}^{\lambda}}{z^{\lambda+1}} d z+(1-\delta)(1-q) \int_{\hat{z}^{\prime}}^{\infty} z^{\eta} \frac{\lambda \hat{z}^{\lambda}}{z^{\lambda+1}} d z \\
& +\left[\delta+(1-\delta) q F_{I}(\bar{z})+(1-\delta)(1-q) F_{I}\left(\hat{z}^{\prime}\right)\right] X_{I}^{\prime} \\
& =\frac{q \int_{\bar{z}}^{\infty}\left(e^{\phi d t} z\right)^{\eta} \frac{\lambda \hat{z}^{\lambda}}{z^{\lambda+1}} d z+(1-q) \int_{\hat{z}^{\prime}}^{\infty} z^{\eta} \frac{\lambda \hat{z}^{\lambda}}{z^{\lambda+1}} d z}{1-q F_{I}(\bar{z})-(1-q) F_{I}\left(\hat{z}^{\prime}\right)} \\
& =\frac{q e^{(\phi-\mu) \eta d t} a^{\lambda-\eta}+(1-q) e^{-\lambda \mu d t}}{q a^{\lambda}+(1-q) e^{-\lambda \mu d t}} e^{\mu \eta d t} \frac{\lambda}{\lambda-\eta} \hat{z}^{\eta}
\end{aligned}
$$

In doing this I am using the exit rate per unit of time, so $\delta$ is indeed the measure of firms that exited the market in the term $d t=1$.

For N-firms we need to keep to track all the endogenous exits from group-I for the 
last $\hat{t}$ periods. Define:

$$
\begin{gathered}
k(\check{z}, d t) \equiv q \int_{\check{z}}^{a \check{z}} e^{\phi \eta d t} z^{\eta} \frac{\lambda \check{z}^{\lambda}}{z^{\lambda+1}} d z+(1-q) \int_{\check{z}}^{e^{\mu d t} \check{z}} z^{\eta} \frac{\lambda \check{z}^{\lambda}}{z^{\lambda+1}} d z \\
k(\check{z}, d t)=q \frac{\lambda}{\lambda-\eta} e^{\phi \eta d t}\left[1-a^{\eta-\lambda}\right] \check{z}^{\eta}+(1-q) \frac{\lambda}{\lambda-\eta}\left[1-e^{(\eta-\lambda) \mu d t}\right] \check{z}^{\eta}
\end{gathered}
$$

Then:

$$
\begin{gathered}
X_{N}=\int_{0}^{\hat{t}} e^{-\delta s} k(\tilde{z}, s) d s \\
=\left(q \frac{\lambda}{\lambda-\eta} e^{\phi \eta \hat{t}}\left[1-a^{\eta-\lambda}\right]+(1-q) \frac{\lambda}{\lambda-\eta}\left[1-e^{(\eta-\lambda) \mu \hat{t}}\right]\right)\left(e^{-\delta \hat{t}} e^{\eta \mu \hat{t}}\right) \tilde{z}^{\eta}
\end{gathered}
$$

Moreover:

$$
\begin{gathered}
e^{\gamma d t}=\frac{w^{\prime}}{w}=\left(\frac{\alpha X_{I}^{\prime}+(1-\alpha) X_{N}^{\prime}}{\alpha X_{I}+(1-\alpha) X_{N}}\right)^{1-\theta}= \\
\left(\frac{\alpha \frac{q e^{(\phi-\mu) \eta d t} a^{\lambda-\eta}+(1-q) e^{-\lambda \mu d t}}{q a^{\lambda}+(1-q) e^{-\lambda \mu d t}} e^{\mu \eta d t}+(1-\alpha)\left(q e^{\phi \eta \hat{t}}\left[1-a^{\eta-\lambda}\right]+(1-q)\left[1-e^{(\eta-\lambda) \mu \hat{t}}\right]\right)\left(e^{-\delta \hat{t}} e^{\eta \mu \hat{t}}\right)}{\alpha e^{\eta \mu d t}+(1-\alpha)\left(q e^{\phi \eta \hat{t}}\left[1-a^{\eta-\lambda}\right]+(1-q)\left[1-e^{(\eta-\lambda) \mu \hat{t}}\right]\right)\left(e^{-\delta \hat{t}} e^{\eta \mu \hat{t}}\right)} e^{\eta \mu d t}\right)^{1-\theta}
\end{gathered}
$$

Since $\gamma=\mu \eta(1-\theta)$ :

$$
q e^{(\phi-\mu) \eta d t} a^{\lambda-\eta}+(1-q) e^{-\lambda \mu d t}=q a^{\lambda}+(1-q) e^{-\lambda \mu d t}
$$

Which gives

$$
e^{(\phi-\mu) d t}=a \leq 1 \Rightarrow \phi \leq \mu
$$

Now we need to show that $\phi \geq \mu$. Remember that in the solution of the optimal innovation policy we ignored the possibility that a successfully innovating firm might also want to sell the technology. An operating I-firm will not exit the market in case of a successful innovation if and only if:

$$
\phi z \geq \hat{z} \mu
$$


We know that this firm is operating now, i.e.:

$$
z \geq \hat{z}
$$

Hence a firm that has just innovated remains in the market if and only if

$$
\phi \geq \mu
$$

Notice that this is a condition that does not depend on productivity, hence if it does not hold, it does not hold for any firm. Suppose that $\phi<\mu$. Then no firm will have the incentive to put resources on innovation, since they will exit the market even though they are lucky and they get to innovate. Then there will not be investment on productivity in the economy. This would imply no growth, giving $\gamma=\mu=1$. This would give contradiction since $\phi \geq 1$.

This shows, together with $\phi \leq \mu$ that $\phi=\mu$.

To prove that the stationary distribution is Pareto if we start with a Pareto distribution

$$
\begin{aligned}
F_{I}^{\prime}(z)= & (1-\delta) q\left[F_{I}\left(\frac{z}{e^{\phi d t}}\right)-F_{I}\left(e^{(\mu-\phi) d t} \hat{z}\right)\right]+ \\
& (1-\delta)(1-q)\left[F_{I}(z)-F_{I}\left(e^{\mu d t} \hat{z}\right)\right]+ \\
& G(z)\left[\delta+(1-\delta)\left(q F_{I}\left(e^{(\mu-\phi) d t} \hat{z}\right)+(1-q) F_{I}\left(e^{\mu d t} \hat{z}\right)\right)\right]
\end{aligned}
$$

Because entrants are drawn from the equilibrium distribution, $G(z)=F_{I}^{\prime}(z)$ and the 
initial distribution is assumed to be Pareto.

$$
\begin{aligned}
& F_{I}^{\prime}(z)=(1-\delta) q\left[\left(1-\left(e^{\phi d t} \frac{\hat{z}}{z}\right)^{\lambda}\right)-F_{I}\left(e^{(\mu-\phi) d t} \hat{z}\right)\right] \\
& (1-\delta)(1-q)\left[1-\left(\frac{\hat{z}}{z}\right)^{\lambda}-F_{I}\left(e^{\mu d t} \hat{z}\right)\right]+ \\
& \left(1-\left(e^{\mu d t} \frac{\hat{z}}{z}\right)^{\lambda}\right)\left[\delta+(1-\delta)\left(q F_{I}\left(e^{(\mu-\phi) d t} \frac{\hat{z}}{\varepsilon}\right)+(1-q) F_{I}\left(e^{\mu d t} \hat{z}\right)\right]\right. \\
& F_{I}^{\prime}(z)=(1-\delta) q\left(1-\left(e^{\phi d t} \frac{\hat{z}}{z}\right)^{\lambda}\right) \\
& -(1-\delta) q\left(e^{\mu d t} \frac{\hat{z}}{z}\right)^{\lambda}\left(1-e^{(\phi-\mu) \lambda d t}\right) \\
& (1-\delta)(1-q)\left[1-\left(\frac{\hat{z}}{z}\right)^{\lambda}\right]-\left(e^{\mu d t} \frac{\hat{z}}{z}\right)^{\lambda}(1-\delta)(1-q)\left(1-\frac{1}{e^{\mu \lambda d t}}\right) \\
& \left(1-\left(e^{\mu d t} \frac{\hat{z}}{z}\right)^{\lambda}\right) \delta \\
& F_{I}^{\prime}(z)=1-\left(e^{\mu d t} \frac{\hat{z}}{z}\right)^{\lambda} a \\
& a=(1-\delta) q e^{(\phi-\mu) \lambda d t}+ \\
& (1-\delta) q\left(1-e^{(\phi-\mu) \lambda d t}\right)+ \\
& (1-\delta)(1-q)\left(\frac{1}{e^{\mu \lambda d t}}+1-\frac{1}{e^{\mu \lambda d t}}\right)+\delta
\end{aligned}
$$

If $\phi-\mu=0$ then $a=1$ which proves the statement.

Proposition 3 (Proof).

Define:

$$
X_{I} \equiv E_{I}\left(z^{\eta}\right)
$$




$$
X_{N} \equiv E_{N}\left(z^{\eta}\right)
$$

Also define the cumulative distribution function for the group $I$ as:

$$
F_{I}(z)= \begin{cases}1-\left(\frac{\hat{z}}{z}\right)^{\lambda}, & \text { if } z \geq \hat{z} \\ 0, & \mathrm{o} / \mathrm{w}\end{cases}
$$

Moreover, let $M_{I}$ and $M_{N}$ be the number of firms in each group, which will be constant in the BGP, let $\alpha \equiv \frac{M_{I}}{M_{I}+M_{N}}$. To show that $\phi=\mu$ we first show that $\mu \leq \phi$ along the BGP. Define $\bar{z} \equiv \max \left\{\hat{z}, e^{\mu-\phi} \hat{z}\right\}$, and $a \equiv \frac{\hat{z}}{\bar{z}}$. Then

$$
\begin{aligned}
X_{I}^{\prime}=(1- & \delta) q \int_{\bar{z}}^{\infty}\left(e^{\phi d t} z\right)^{\eta} \frac{\lambda \tilde{z}^{\lambda}}{z^{\lambda+1}} d z+(1-\delta)(1-q) \int_{\hat{z}^{\prime}}^{\infty} z^{\eta} \frac{\lambda \hat{z}^{\lambda}}{z^{\lambda+1}} d z \\
& +\left[\delta+(1-\delta) q F_{I}(\bar{z})+(1-\delta)(1-q) F_{I}\left(\hat{z}^{\prime}\right)\right] X_{I}^{\prime} \\
& =\frac{q \int_{\bar{z}}^{\infty}\left(e^{\phi d t} z\right)^{\eta} \frac{\lambda \hat{z}^{\lambda}}{z^{\lambda+1}} d z+(1-q) \int_{\hat{z}^{\prime}}^{\infty} z^{\eta} \frac{\lambda \hat{z}^{\lambda}}{z^{\lambda+1}} d z}{1-q F_{I}(\bar{z})-(1-q) F_{I}\left(\hat{z}^{\prime}\right)} \\
& =\frac{q e^{(\phi-\mu) \eta d t} a^{\lambda-\eta}+(1-q) e^{-\lambda \mu d t}}{q a^{\lambda}+(1-q) e^{-\lambda \mu d t}} e^{\mu \eta d t} \frac{\lambda}{\lambda-\eta} \hat{z}^{\eta}
\end{aligned}
$$

In doing this I am using the exit rate per unit of time, so $\delta$ is indeed the measure of firms that exited the market in the term $d t=1$.

For N-firms we need to keep to track all the endogenous exits from group-I for the last $\hat{t}$ periods. Define:

$$
\begin{gathered}
k(\check{z}, d t) \equiv q \int_{\check{z}}^{a \check{z}} e^{\phi \eta d t} z^{\eta} \frac{\lambda \check{z}^{\lambda}}{z^{\lambda+1}} d z+(1-q) \int_{\check{z}}^{e^{\mu d t} \check{z}} z^{\eta} \frac{\lambda \check{z}^{\lambda}}{z^{\lambda+1}} d z \\
k(\check{z}, d t)=q \frac{\lambda}{\lambda-\eta} e^{\phi \eta d t}\left[1-a^{\eta-\lambda}\right] \check{z}^{\eta}+(1-q) \frac{\lambda}{\lambda-\eta}\left[1-e^{(\eta-\lambda) \mu d t}\right] \check{z}^{\eta}
\end{gathered}
$$

Then:

$$
X_{N}=\int_{0}^{\hat{t}} e^{-\delta s} k(\tilde{z}, s) d s
$$




$$
=\left(q \frac{\lambda}{\lambda-\eta} e^{\phi \eta \hat{t}}\left[1-a^{\eta-\lambda}\right]+(1-q) \frac{\lambda}{\lambda-\eta}\left[1-e^{(\eta-\lambda) \mu \hat{t}}\right]\right)\left(e^{-\delta \hat{t}} e^{\eta \mu \hat{t}}\right) \tilde{z}^{\eta}
$$

Moreover:

$$
\begin{gathered}
e^{\gamma d t}=\frac{w^{\prime}}{w}=\left(\frac{\alpha X_{I}^{\prime}+(1-\alpha) X_{N}^{\prime}}{\alpha X_{I}+(1-\alpha) X_{N}}\right)^{1-\theta}= \\
\left(\frac{\alpha \frac{q e^{(\phi-\mu) \eta d t} a^{\lambda-\eta}+(1-q) e^{-\lambda \mu d t}}{q a^{\lambda}+(1-q) e^{-\lambda \mu d t}} e^{\mu \eta d t}+(1-\alpha)\left(q e^{\phi \eta \hat{t}}\left[1-a^{\eta-\lambda}\right]+(1-q)\left[1-e^{(\eta-\lambda) \mu \hat{t}}\right]\right)\left(e^{-\delta \hat{t}} e^{\eta \mu \hat{t}}\right)}{\alpha e^{\eta \mu d t}+(1-\alpha)\left(q e^{\phi \eta \hat{t}}\left[1-a^{\eta-\lambda}\right]+(1-q)\left[1-e^{(\eta-\lambda) \mu \hat{t}}\right]\right)\left(e^{-\delta \hat{t}} e^{\eta \mu \hat{t}}\right)} e^{\eta \mu d t}\right)^{1-\theta}
\end{gathered}
$$

Since $\gamma=\mu \eta(1-\theta)$ :

$$
q e^{(\phi-\mu) \eta d t} a^{\lambda-\eta}+(1-q) e^{-\lambda \mu d t}=q a^{\lambda}+(1-q) e^{-\lambda \mu d t}
$$

Which gives

$$
e^{(\phi-\mu) d t}=a \leq 1 \Rightarrow \phi \leq \mu
$$

Now we need to show that $\phi \geq \mu$. Remember that in the solution of the optimal innovation policy we ignored the possibility that a successfully innovating firm might also want to sell the technology. An operating I-firm will not exit the market in case of a successful innovation if and only if:

$$
\phi z \geq \hat{z} \mu
$$

We know that this firm is operating now, i.e.:

$$
z \geq \hat{z}
$$

Hence a firm that has just innovated remains in the market if and only if

$$
\phi \geq \mu
$$


Notice that this is a condition that does not depend on productivity, hence if it does not hold, it does not hold for any firm. Suppose that $\phi<\mu$. Then no firm will have the incentive to put resources on innovation, since they will exit the market even though they are lucky and they get to innovate. Then there will not be investment on productivity in the economy. This would imply no growth, giving $\gamma=\mu=1$. This would give contradiction since $\phi \geq 1$.

This shows, together with $\phi \leq \mu$ that $\phi=\mu$.

To prove that the stationary distribution is Pareto if we start with a Pareto distribution

$$
\begin{aligned}
F_{I}^{\prime}(z)= & (1-\delta) q\left[F_{I}\left(\frac{z}{e^{\phi d t}}\right)-F_{I}\left(e^{(\mu-\phi) d t} \hat{z}\right)\right]+ \\
& (1-\delta)(1-q)\left[F_{I}(z)-F_{I}\left(e^{\mu d t} \hat{z}\right)\right]+ \\
& G(z)\left[\delta+(1-\delta)\left(q F_{I}\left(e^{(\mu-\phi) d t} \hat{z}\right)+(1-q) F_{I}\left(e^{\mu d t} \hat{z}\right)\right)\right]
\end{aligned}
$$

Because entrants are drawn from the equilibrium distribution, $G(z)=F_{I}^{\prime}(z)$ and the initial distribution is assumed to be Pareto.

$$
\begin{aligned}
F_{I}^{\prime}(z)= & (1-\delta) q\left[\left(1-\left(e^{\phi d t} \frac{\hat{z}}{z}\right)^{\lambda}\right)-F_{I}\left(e^{(\mu-\phi) d t} \hat{z}\right)\right] \\
& (1-\delta)(1-q)\left[1-\left(\frac{\hat{z}}{z}\right)^{\lambda}-F_{I}\left(e^{\mu d t} \hat{z}\right)\right]+ \\
& \left(1-\left(e^{\mu d t} \frac{\hat{z}}{z}\right)^{\lambda}\right)\left[\delta+(1-\delta)\left(q F_{I}\left(e^{(\mu-\phi) d t} \frac{\hat{z}}{\varepsilon}\right)+(1-q) F_{I}\left(e^{\mu d t} \hat{z}\right)\right]\right.
\end{aligned}
$$




$$
\begin{aligned}
& F_{I}^{\prime}(z)=(1-\delta) q\left(1-\left(e^{\phi d t} \frac{\hat{z}}{z}\right)^{\lambda}\right) \\
&-(1-\delta) q\left(e^{\mu d t} \frac{\hat{z}}{z}\right)^{\lambda}\left(1-e^{(\phi-\mu) \lambda d t}\right) \\
&(1-\delta)(1-q)\left[1-\left(\frac{\hat{z}}{z}\right)^{\lambda}\right]-\left(e^{\mu d t} \frac{\hat{z}}{z}\right)^{\lambda}(1-\delta)(1-q)\left(1-\frac{1}{e^{\mu \lambda d t}}\right) \\
&\left(1-\left(e^{\mu d t} \frac{\hat{z}}{z}\right)^{\lambda}\right) \delta \\
& F_{I}^{\prime}(z)=1-\left(e^{\mu d t} \frac{\hat{z}}{z}\right)^{\lambda} a \\
& a \quad(1-\delta) q e^{(\phi-\mu) \lambda d t}+ \\
&(1-\delta) q\left(1-e^{(\phi-\mu) \lambda d t}\right)+ \\
&(1-\delta)(1-q)\left(\frac{1}{e^{\mu \lambda d t}}+1-\frac{1}{e^{\mu \lambda d t}}\right)+\delta
\end{aligned}
$$

If $\phi-\mu=0$ then $a=1$ which proves the statement.

\section{Proposition 3 (Proof).}

Define:

$$
\begin{gathered}
X_{I} \equiv E_{I}\left(z^{\eta}\right) \\
X_{N} \equiv E_{N}\left(z^{\eta}\right)
\end{gathered}
$$

Also define the cumulative distribution function for the group $I$ as:

$$
F_{I}(z)= \begin{cases}1-\left(\frac{\hat{z}}{z}\right)^{\lambda}, & \text { if } z \geq \hat{z} \\ 0, & \text { o/w }\end{cases}
$$

Moreover, let $M_{I}$ and $M_{N}$ be the number of firms in each group, which will be constant in the BGP, let $\alpha \equiv \frac{M_{I}}{M_{I}+M_{N}}$. To show that $\phi=\mu$ we first show that $\mu \leq \phi$ 
along the BGP. Define $\bar{z} \equiv \max \left\{\hat{z}, e^{\mu-\phi} \hat{z}\right\}$, and $a \equiv \frac{\hat{z}}{\bar{z}}$. Then

$$
\begin{aligned}
X_{I}^{\prime}=(1- & \delta) q \int_{\bar{z}}^{\infty}\left(e^{\phi d t} z\right)^{\eta} \frac{\lambda \tilde{z}^{\lambda}}{z^{\lambda+1}} d z+(1-\delta)(1-q) \int_{\hat{z}^{\prime}}^{\infty} z^{\eta} \frac{\lambda \hat{z}^{\lambda}}{z^{\lambda+1}} d z \\
& +\left[\delta+(1-\delta) q F_{I}(\bar{z})+(1-\delta)(1-q) F_{I}\left(\hat{z}^{\prime}\right)\right] X_{I}^{\prime} \\
& =\frac{q \int_{\bar{z}}^{\infty}\left(e^{\phi d t} z\right)^{\eta} \frac{\lambda \hat{z}^{\lambda}}{z^{\lambda+1}} d z+(1-q) \int_{\hat{z}^{\prime}}^{\infty} z^{\eta} \frac{\lambda \hat{z}^{\lambda}}{z^{\lambda+1}} d z}{1-q F_{I}(\bar{z})-(1-q) F_{I}\left(\hat{z}^{\prime}\right)} \\
& =\frac{q e^{(\phi-\mu) \eta d t} a^{\lambda-\eta}+(1-q) e^{-\lambda \mu d t}}{q a^{\lambda}+(1-q) e^{-\lambda \mu d t}} e^{\mu \eta d t} \frac{\lambda}{\lambda-\eta} \hat{z}^{\eta}
\end{aligned}
$$

In doing this I am using the exit rate per unit of time, so $\delta$ is indeed the measure of firms that exited the market in the term $d t=1$.

For N-firms we need to keep to track all the endogenous exits from group-I for the last $\hat{t}$ periods. Define:

$$
\begin{gathered}
k(\check{z}, d t) \equiv q \int_{\check{z}}^{a \check{z}} e^{\phi \eta d t} z^{\eta} \frac{\lambda \check{z}^{\lambda}}{z^{\lambda+1}} d z+(1-q) \int_{\check{z}}^{e^{\mu d t} \check{z}} z^{\eta} \frac{\lambda \check{z}^{\lambda}}{z^{\lambda+1}} d z \\
k(\check{z}, d t)=q \frac{\lambda}{\lambda-\eta} e^{\phi \eta d t}\left[1-a^{\eta-\lambda}\right] \check{z}^{\eta}+(1-q) \frac{\lambda}{\lambda-\eta}\left[1-e^{(\eta-\lambda) \mu d t}\right] \check{z}^{\eta}
\end{gathered}
$$

Then:

$$
\begin{gathered}
X_{N}=\int_{0}^{\hat{t}} e^{-\delta s} k(\tilde{z}, s) d s \\
=\left(q \frac{\lambda}{\lambda-\eta} e^{\phi \eta \hat{t}}\left[1-a^{\eta-\lambda}\right]+(1-q) \frac{\lambda}{\lambda-\eta}\left[1-e^{(\eta-\lambda) \mu \hat{t}}\right]\right)\left(e^{-\delta \hat{t}} e^{\eta \mu \hat{t}}\right) \tilde{z}^{\eta}
\end{gathered}
$$

Moreover:

$$
\begin{gathered}
e^{\gamma d t}=\frac{w^{\prime}}{w}=\left(\frac{\alpha X_{I}^{\prime}+(1-\alpha) X_{N}^{\prime}}{\alpha X_{I}+(1-\alpha) X_{N}}\right)^{1-\theta}= \\
\left(\frac{\alpha \frac{q e^{(\phi-\mu) \eta d t} a^{\lambda-\eta}+(1-q) e^{-\lambda \mu d t}}{q a^{\lambda}+(1-q) e^{-\lambda \mu d t}} e^{\mu \eta d t}+(1-\alpha)\left(q e^{\phi \eta \hat{t}}\left[1-a^{\eta-\lambda}\right]+(1-q)\left[1-e^{(\eta-\lambda) \mu \hat{t}}\right]\right)\left(e^{-\delta \hat{t}} e^{\eta \mu \hat{t}}\right)}{\alpha e^{\eta \mu d t}+(1-\alpha)\left(q e^{\phi \eta \hat{t}}\left[1-a^{\eta-\lambda}\right]+(1-q)\left[1-e^{(\eta-\lambda) \mu \hat{t}}\right]\right)\left(e^{-\delta \hat{t}} e^{\eta \mu \hat{t}}\right)} e^{\eta \mu d t}\right)^{1-\theta}
\end{gathered}
$$


Since $\gamma=\mu \eta(1-\theta)$ :

$$
q e^{(\phi-\mu) \eta d t} a^{\lambda-\eta}+(1-q) e^{-\lambda \mu d t}=q a^{\lambda}+(1-q) e^{-\lambda \mu d t}
$$

Which gives

$$
e^{(\phi-\mu) d t}=a \leq 1 \Rightarrow \phi \leq \mu
$$

Now we need to show that $\phi \geq \mu$. Remember that in the solution of the optimal innovation policy we ignored the possibility that a successfully innovating firm might also want to sell the technology. An operating I-firm will not exit the market in case of a successful innovation if and only if:

$$
\phi z \geq \hat{z} \mu
$$

We know that this firm is operating now, i.e.:

$$
z \geq \hat{z}
$$

Hence a firm that has just innovated remains in the market if and only if

$$
\phi \geq \mu
$$

Notice that this is a condition that does not depend on productivity, hence if it does not hold, it does not hold for any firm. Suppose that $\phi<\mu$. Then no firm will have the incentive to put resources on innovation, since they will exit the market even though they are lucky and they get to innovate. Then there will not be investment on productivity in the economy. This would imply no growth, giving $\gamma=\mu=1$. This would give contradiction since $\phi \geq 1$.

This shows, together with $\phi \leq \mu$ that $\phi=\mu$.

To prove that the stationary distribution is Pareto if we start with a Pareto distri- 
bution

$$
\begin{aligned}
F_{I}^{\prime}(z)= & (1-\delta) q\left[F_{I}\left(\frac{z}{e^{\phi d t}}\right)-F_{I}\left(e^{(\mu-\phi) d t} \hat{z}\right)\right]+ \\
& (1-\delta)(1-q)\left[F_{I}(z)-F_{I}\left(e^{\mu d t} \hat{z}\right)\right]+ \\
& G(z)\left[\delta+(1-\delta)\left(q F_{I}\left(e^{(\mu-\phi) d t} \hat{z}\right)+(1-q) F_{I}\left(e^{\mu d t} \hat{z}\right)\right)\right]
\end{aligned}
$$

Because entrants are drawn from the equilibrium distribution, $G(z)=F_{I}^{\prime}(z)$ and the initial distribution is assumed to be Pareto.

$$
\begin{aligned}
& F_{I}^{\prime}(z)=(1-\delta) q\left[\left(1-\left(e^{\phi d t} \frac{\hat{z}}{z}\right)^{\lambda}\right)-F_{I}\left(e^{(\mu-\phi) d t} \hat{z}\right)\right] \\
& (1-\delta)(1-q)\left[1-\left(\frac{\hat{z}}{z}\right)^{\lambda}-F_{I}\left(e^{\mu d t} \hat{z}\right)\right]+ \\
& \left(1-\left(e^{\mu d t} \frac{\hat{z}}{z}\right)^{\lambda}\right)\left[\delta+(1-\delta)\left(q F_{I}\left(e^{(\mu-\phi) d t} \frac{\hat{z}}{\varepsilon}\right)+(1-q) F_{I}\left(e^{\mu d t} \hat{z}\right)\right]\right. \\
& F_{I}^{\prime}(z)=(1-\delta) q\left(1-\left(e^{\phi d t} \frac{\hat{z}}{z}\right)^{\lambda}\right) \\
& -(1-\delta) q\left(e^{\mu d t} \frac{\hat{z}}{z}\right)^{\lambda}\left(1-e^{(\phi-\mu) \lambda d t}\right) \\
& (1-\delta)(1-q)\left[1-\left(\frac{\hat{z}}{z}\right)^{\lambda}\right]-\left(e^{\mu d t} \frac{\hat{z}}{z}\right)^{\lambda}(1-\delta)(1-q)\left(1-\frac{1}{e^{\mu \lambda d t}}\right) \\
& \left(1-\left(e^{\mu d t} \frac{\hat{z}}{z}\right)^{\lambda}\right) \delta \\
& F_{I}^{\prime}(z)=1-\left(e^{\mu d t} \frac{\hat{z}}{z}\right)^{\lambda} a
\end{aligned}
$$




$$
\begin{aligned}
a= & (1-\delta) q e^{(\phi-\mu) \lambda d t}+ \\
& (1-\delta) q\left(1-e^{(\phi-\mu) \lambda d t}\right)+ \\
& (1-\delta)(1-q)\left(\frac{1}{e^{\mu \lambda d t}}+1-\frac{1}{e^{\mu \lambda d t}}\right)+\delta
\end{aligned}
$$

If $\phi-\mu=0$ then $a=1$ which proves the statement.

\section{Proposition 3 (Proof).}

Define:

$$
\begin{gathered}
X_{I} \equiv E_{I}\left(z^{\eta}\right) \\
X_{N} \equiv E_{N}\left(z^{\eta}\right)
\end{gathered}
$$

Also define the cumulative distribution function for the group $I$ as:

$$
F_{I}(z)= \begin{cases}1-\left(\frac{\hat{z}}{z}\right)^{\lambda}, & \text { if } z \geq \hat{z} \\ 0, & \mathrm{o} / \mathrm{w}\end{cases}
$$

Moreover, let $M_{I}$ and $M_{N}$ be the number of firms in each group, which will be constant in the BGP, let $\alpha \equiv \frac{M_{I}}{M_{I}+M_{N}}$. To show that $\phi=\mu$ we first show that $\mu \leq \phi$ along the BGP. Define $\bar{z} \equiv \max \left\{\hat{z}, e^{\mu-\phi} \hat{z}\right\}$, and $a \equiv \frac{\hat{z}}{\bar{z}}$. Then

$$
\begin{aligned}
X_{I}^{\prime}=(1- & \delta) q \int_{\bar{z}}^{\infty}\left(e^{\phi d t} z\right)^{\eta} \frac{\lambda \tilde{z}^{\lambda}}{z^{\lambda+1}} d z+(1-\delta)(1-q) \int_{\hat{z}^{\prime}}^{\infty} z^{\eta} \frac{\lambda \hat{z}^{\lambda}}{z^{\lambda+1}} d z \\
& +\left[\delta+(1-\delta) q F_{I}(\bar{z})+(1-\delta)(1-q) F_{I}\left(\hat{z}^{\prime}\right)\right] X_{I}^{\prime} \\
& =\frac{q \int_{\bar{z}}^{\infty}\left(e^{\phi d t} z\right)^{\eta} \frac{\lambda \hat{z}^{\lambda}}{z^{\lambda+1}} d z+(1-q) \int_{\hat{z}^{\prime}}^{\infty} z^{\eta} \frac{\lambda \hat{z}^{\lambda}}{z^{\lambda+1}} d z}{1-q F_{I}(\bar{z})-(1-q) F_{I}\left(\hat{z}^{\prime}\right)} \\
& =\frac{q e^{(\phi-\mu) \eta d t} a^{\lambda-\eta}+(1-q) e^{-\lambda \mu d t}}{q a^{\lambda}+(1-q) e^{-\lambda \mu d t}} e^{\mu \eta d t} \frac{\lambda}{\lambda-\eta} \hat{z}^{\eta}
\end{aligned}
$$

In doing this I am using the exit rate per unit of time, so $\delta$ is indeed the measure of firms that exited the market in the term $d t=1$.

For N-firms we need to keep to track all the endogenous exits from group-I for the 
last $\hat{t}$ periods. Define:

$$
\begin{gathered}
k(\check{z}, d t) \equiv q \int_{\check{z}}^{a \check{z}} e^{\phi \eta d t} z^{\eta} \frac{\lambda \check{z}^{\lambda}}{z^{\lambda+1}} d z+(1-q) \int_{\check{z}}^{e^{\mu d t} \check{z}} z^{\eta} \frac{\lambda \check{z}^{\lambda}}{z^{\lambda+1}} d z \\
k(\check{z}, d t)=q \frac{\lambda}{\lambda-\eta} e^{\phi \eta d t}\left[1-a^{\eta-\lambda}\right] \check{z}^{\eta}+(1-q) \frac{\lambda}{\lambda-\eta}\left[1-e^{(\eta-\lambda) \mu d t}\right] \check{z}^{\eta}
\end{gathered}
$$

Then:

$$
\begin{gathered}
X_{N}=\int_{0}^{\hat{t}} e^{-\delta s} k(\tilde{z}, s) d s \\
=\left(q \frac{\lambda}{\lambda-\eta} e^{\phi \eta \hat{t}}\left[1-a^{\eta-\lambda}\right]+(1-q) \frac{\lambda}{\lambda-\eta}\left[1-e^{(\eta-\lambda) \mu \hat{t}}\right]\right)\left(e^{-\delta \hat{t}} e^{\eta \mu \hat{t}}\right) \tilde{z}^{\eta}
\end{gathered}
$$

Moreover:

$$
\begin{gathered}
e^{\gamma d t}=\frac{w^{\prime}}{w}=\left(\frac{\alpha X_{I}^{\prime}+(1-\alpha) X_{N}^{\prime}}{\alpha X_{I}+(1-\alpha) X_{N}}\right)^{1-\theta}= \\
\left(\frac{\alpha \frac{q e^{(\phi-\mu) \eta d t} a^{\lambda-\eta}+(1-q) e^{-\lambda \mu d t}}{q a^{\lambda}+(1-q) e^{-\lambda \mu d t}} e^{\mu \eta d t}+(1-\alpha)\left(q e^{\phi \eta \hat{t}}\left[1-a^{\eta-\lambda}\right]+(1-q)\left[1-e^{(\eta-\lambda) \mu \hat{t}}\right]\right)\left(e^{-\delta \hat{t}} e^{\eta \mu \hat{t}}\right)}{\alpha e^{\eta \mu d t}+(1-\alpha)\left(q e^{\phi \eta \hat{t}}\left[1-a^{\eta-\lambda}\right]+(1-q)\left[1-e^{(\eta-\lambda) \mu \hat{t}}\right]\right)\left(e^{-\delta \hat{t}} e^{\eta \mu \hat{t}}\right)} e^{\eta \mu d t}\right)^{1-\theta}
\end{gathered}
$$

Since $\gamma=\mu \eta(1-\theta)$ :

$$
q e^{(\phi-\mu) \eta d t} a^{\lambda-\eta}+(1-q) e^{-\lambda \mu d t}=q a^{\lambda}+(1-q) e^{-\lambda \mu d t}
$$

Which gives

$$
e^{(\phi-\mu) d t}=a \leq 1 \Rightarrow \phi \leq \mu
$$

Now we need to show that $\phi \geq \mu$. Remember that in the solution of the optimal innovation policy we ignored the possibility that a successfully innovating firm might also want to sell the technology. An operating I-firm will not exit the market in case of a successful innovation if and only if:

$$
\phi z \geq \hat{z} \mu
$$


We know that this firm is operating now, i.e.:

$$
z \geq \hat{z}
$$

Hence a firm that has just innovated remains in the market if and only if

$$
\phi \geq \mu
$$

Notice that this is a condition that does not depend on productivity, hence if it does not hold, it does not hold for any firm. Suppose that $\phi<\mu$. Then no firm will have the incentive to put resources on innovation, since they will exit the market even though they are lucky and they get to innovate. Then there will not be investment on productivity in the economy. This would imply no growth, giving $\gamma=\mu=1$. This would give contradiction since $\phi \geq 1$.

This shows, together with $\phi \leq \mu$ that $\phi=\mu$.

To prove that the stationary distribution is Pareto if we start with a Pareto distribution

$$
\begin{aligned}
F_{I}^{\prime}(z)= & (1-\delta) q\left[F_{I}\left(\frac{z}{e^{\phi d t}}\right)-F_{I}\left(e^{(\mu-\phi) d t} \hat{z}\right)\right]+ \\
& (1-\delta)(1-q)\left[F_{I}(z)-F_{I}\left(e^{\mu d t} \hat{z}\right)\right]+ \\
& G(z)\left[\delta+(1-\delta)\left(q F_{I}\left(e^{(\mu-\phi) d t} \hat{z}\right)+(1-q) F_{I}\left(e^{\mu d t} \hat{z}\right)\right)\right]
\end{aligned}
$$

Because entrants are drawn from the equilibrium distribution, $G(z)=F_{I}^{\prime}(z)$ and the 
initial distribution is assumed to be Pareto.

$$
\begin{aligned}
& F_{I}^{\prime}(z)=(1-\delta) q\left[\left(1-\left(e^{\phi d t} \frac{\hat{z}}{z}\right)^{\lambda}\right)-F_{I}\left(e^{(\mu-\phi) d t} \hat{z}\right)\right] \\
& (1-\delta)(1-q)\left[1-\left(\frac{\hat{z}}{z}\right)^{\lambda}-F_{I}\left(e^{\mu d t} \hat{z}\right)\right]+ \\
& \left(1-\left(e^{\mu d t} \frac{\hat{z}}{z}\right)^{\lambda}\right)\left[\delta+(1-\delta)\left(q F_{I}\left(e^{(\mu-\phi) d t} \frac{\hat{z}}{\varepsilon}\right)+(1-q) F_{I}\left(e^{\mu d t} \hat{z}\right)\right]\right. \\
& F_{I}^{\prime}(z)=(1-\delta) q\left(1-\left(e^{\phi d t} \frac{\hat{z}}{z}\right)^{\lambda}\right) \\
& -(1-\delta) q\left(e^{\mu d t} \frac{\hat{z}}{z}\right)^{\lambda}\left(1-e^{(\phi-\mu) \lambda d t}\right) \\
& (1-\delta)(1-q)\left[1-\left(\frac{\hat{z}}{z}\right)^{\lambda}\right]-\left(e^{\mu d t} \frac{\hat{z}}{z}\right)^{\lambda}(1-\delta)(1-q)\left(1-\frac{1}{e^{\mu \lambda d t}}\right) \\
& \left(1-\left(e^{\mu d t} \frac{\hat{z}}{z}\right)^{\lambda}\right) \delta \\
& F_{I}^{\prime}(z)=1-\left(e^{\mu d t} \frac{\hat{z}}{z}\right)^{\lambda} a \\
& a=(1-\delta) q e^{(\phi-\mu) \lambda d t}+ \\
& (1-\delta) q\left(1-e^{(\phi-\mu) \lambda d t}\right)+ \\
& (1-\delta)(1-q)\left(\frac{1}{e^{\mu \lambda d t}}+1-\frac{1}{e^{\mu \lambda d t}}\right)+\delta
\end{aligned}
$$

If $\phi-\mu=0$ then $a=1$ which proves the statement. 